\title{
Could lymphatic mapping and sentinel node biopsy provide oncological providence for local resectional techniques for colon cancer? A review of the literature Ronan A Cahill*, Joel Leroy and Jacques Marescaux
}

Address: Department of Surgery, IRCAD/EITS, Strasbourg, France

Email: Ronan A Cahill* - cahillra@gmail.com; Joel Leroy - joel.leroy@ircad.fr; Jacques Marescaux - jacques.marescaux@ircad.fr

* Corresponding author

Published: 24 September 2008

BMC Surgery 2008, 8:17 doi:10.1 186/147|-2482-8-17
Received: 22 May 2008

Accepted: 24 September 2008

This article is available from: http://www.biomedcentral.com/I47/-2482/8/17

(c) 2008 Cahill et al; licensee BioMed Central Ltd.

This is an Open Access article distributed under the terms of the Creative Commons Attribution License (http://creativecommons.org/licenses/by/2.0), which permits unrestricted use, distribution, and reproduction in any medium, provided the original work is properly cited.

\begin{abstract}
Background: Endoscopic resectional techniques for colon cancer are undermined by their inability to determine lymph node status. This limits their application to only those lesions at the most minimal risk of lymphatic dissemination whereas their technical capacity could allow intraluminal or even transluminal address of larger lesions. Sentinel node biopsy may theoretically address this breach although the variability of its reported results for this disease is worrisome.
\end{abstract}

Methods: Medline, EMBASE and Cochrane databases were interrogated back to 1999 to identify all publications concerning lymphatic mapping for colon cancer with reference cross-checking for completeness. All reports were examined from the perspective of in vivo technique accuracy selectively in early stage disease (i.e. lesions potentially within the technical capacity of endoscopic resection).

Results: Fifty-two studies detailing the experiences of 3390 patients were identified. Considerable variation in patient characteristics as well as in surgical and histological quality assurances were however evident among the studies identified. In addition, considerable contamination of the studies by inclusion of rectal cancer without subgroup separation was frequent. Indeed such is the heterogeneity of the publications to date, formal meta-analysis to pool patient cohorts in order to definitively ascertain technique accuracy in those with $\mathrm{TI}$ and/or $\mathrm{T} 2$ cancer is not possible. Although lymphatic mapping in early stage neoplasia alone has rarely been specifically studied, those studies that included examination of false negative rates identified high T3/4 patient proportions and larger tumor size as being important confounders. Under selected circumstances however the technique seems to perform sufficiently reliably to allow it prompt consideration of its use to tailor operative extent.

Conclusion: The specific question of whether sentinel node biopsy can augment the oncological propriety for endoscopic resective techniques (including Natural Orifice Transluminal Endoscopic Surgery [NOTES]) cannot be definitively answered at present. Study heterogeneity may account for the variability evident in the results from different centers. Enhanced capacity (perhaps to the level necessary to consider selective avoidance of en bloc mesenteric resection) by its confinement to only early stage disease is plausible although not proven. Specific study of the technique in early stage tumors is clearly essential before proffering this approach. 


\section{Background}

Advances in technological capability have made feasible the local resection of small colonic tumors by intraluminal and even transluminal endoscopy [1-4]. Although primarily now proposed for supposed benign lesions, in concept, selected germinal cancers could also be resected by these means. However, the insensitivity of preoperative radiological imaging for the detection of nodal metastases (approximately $70 \%$ of tumor-containing nodes are less than $5 \mathrm{~mm}$ in size [5-9]) and the inability of biopsy analysis to truly reflect the metastatic potential of the primary means that localized resection of the primary for even the earliest cancers risks either the understaging of systemic disease or the rendering of the effort redundant if formal resection becomes indicated by the full pathology of the resected specimen[10]. A reliable means of definitively establishing lymph node status peroperatively, other than en bloc mesenteric resection, would greatly increase the oncological providence of these techniques and could expand their application.

Sentinel node biopsy would seem on first principles well suited to address this breach as it fulfills a similar role in tumors of the breast and skin. This technique has also been recently proposed to accompany endoscopic dissection of early gastric cancers in order to enhance functional outcome by minimizing the extent of surgical resection [11-13]. Adjoining such a 'diagnostic laparoscopy' to an endoscopic resective technique could be justified in selected patients if the outcome of the node biopsy would permit localized excision as the definitive intervention in place of radical operation. Conversely, if the node is revealed as positive for metastases, the surgeon can confidently advocate radical operation in cases when the tumor appears confined. Synchronous laparoscopy has indeed already been advocated for the endoscopic resection of certain difficult or large polyps[14]. Furthermore, it seems likely that increasing experience with transluminal peritoneal access and intervention (i.e. Natural Orifice Transluminal Endoscopic Surgery [NOTES]) could mean that selective lymph node biopsy without abdominal wall ingress will be practicable in the near future[15]. However, lymphatic mapping in intestinal cancers is still considered controversial because of reports of varying accuracy and concerns regarding reliability and reproducibility.

To date however no comprehensive study or review has been performed from the perspective of using lymphatic mapping to facilitate minimally resective techniques for early stage colon tumors. Analyses to date have instead focused primarily on the capacity of the technique to predict recurrence risk through the upstaging of conventionally node negative disease after standard operation has been performed [16-24]. The main focus has therefore been on Stage II rather than Stage I cancers with the sentinel node biopsy and analysis being performed in addition to rather than in place of formal lymphadenectomy[25]. The aim of this review is therefore to formally interrogate the evidence base in its entirety to determine whether lymphatic mapping can, on any basis, be rationally proposed to augment the oncological propriety of localized endoscopic resection specifically for the small, early stage colon cancers that lie within its scope.

Note: Rectal cancers lie outside the premise of this review as the anatomical arrangement of the mesorectum (specifically its bulk, retroperitoneal position and lack of serosal layer) precludes against intraoperative nodal biopsy for rectal cancer. Furthermore, violation of the mesorectum may also compromise any subsequent attempt at formal oncological resection (and hence negatively impact upon patient outcome) should this be indicated by the pathology of the resected specimen.

\section{Methods}

\section{Search methods for identification of relevant studies}

The following strategy was used to identify relevant publications regarding experience with lymphatic mapping in human patients with colon cancer (see Table 1). Firstly, PubMed software was used to search the Medline database

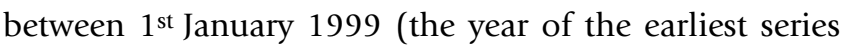
published on the technique in colon cancer) and the $30^{\text {th }}$ July 2008. The Cochrane library (including the Cochrane Central Register of Controlled Trials) and EMBASE databases were also directly searched in a similar fashion. The following expanded Medical Subject Headings were used'sentinel node', 'lymphatic mapping', 'colon cancer', 'colon tumo(u)rs', 'colorectal cancer/tumo(u)rs', 'large intestine' and 'gastrointestinal' (although the intent of the study was confined to colon cancer the later three search terms were included for completeness as some series report mixed cohorts). The reference list of all full publications so identified along with that of consensus papers, review articles, editorials and relevant book chapters were cross-checked for additional relevant publications. Data contained in meeting abstracts were not studied as these were judged unlikely to present sufficient detail for extraction required by our study protocol. Only English language publications were included (language bias however seems unlikely since the literature search in Pubmed and

Table I: Inclusion criteria for inclusion of publication in this review.

\section{Inclusion criteria}

English language publication

Full publication between Ist January 1999 and July 30th 2008.

Human patients with colon cancer

In vivo mapping and node identification 
Embase did not reveal any substantial non-english reports). Finally only those studies that used vivo methodology alone for both the injection and the identification of salient sentinel nodes were analyzed (ex vivo mapping can only be reliably performed if a specimen with its complete mesentery has already been resected intact while ex vivo identification can predispose to false negative glands as redistribution of the dye along the lymphatic chain can occur in the time between injection and node harvest). Intraoperative marking of the sentinel node rather than actual excisional biopsy allowed inclusion however as clearly the intent and purpose is the same.

\section{Assessment of methodological quality of the studies identified}

Each of the studies identified was analyzed for suitability for inclusion according to the criteria laid down by QUADRAS - an evidenced base tool for the assessment of the quality of diagnostic assessment studies[26,27]. By this means it was determined whether the publication was of sufficient quality to allow inclusion in this review.

\section{Data extraction from included studies}

All data extraction was performed by two authors (RAC and JL) with cross-checking to ensure validation. When any disparity or disagreements arose the investigators met with the third author JM as final adjudicator to resolve the debate. The fields for data capture were pre-specified before analysis of the studies identified. Data pertaining to patient demographics, technique methodology (including both surgeon experience as well as precise technical details) and sentinel node efficacy by binary classification (i.e. detection, accuracy, sensitivity and false negative rates as well as negative predictive values) for patients with colon cancer undergoing lymphatic mapping were prepared on Microsoft Excel datasheets. Data from papers that explicitly declared themselves further sub-analyses of a previous study were included with the prior publication. Studies from the same authors but which did not declare themselves to contain overlapping cohorts were analyzed separately although are flagged in the subsequent tables to indicate that this is possibility. When quantitative results were not presented and were not extractable only that data that was useful to this analysis was extracted (data not presented and impossible to extract is denoted in the tables by ns (i.e. 'not stated'). Otherwise the paper was excluded. In cases of mixed populations, where possible, only data relating to the in vivo mapping and biopsy of sentinel nodes in colon cancer were extracted. If not possible, the data was included with note made of the circumstances. Finally any further formal results analyses or additional hard data (rather than speculative commentary or theoretical opinion) from the Methods, Results or Discussions sections of the studies was also recorded to allow for subsequent analysis and consideration.

\section{Sentinel Node Performance Parameters}

Where possible, $2 \times 2$ contingency tables were built comprising true positive (both sentinel and non-sentinel nodes involved), true negative (both sentinel and nonsentinel nodes clear), false negative (sentinel node clear but non-sentinel nodes involved). The term 'false positive' (and hence calculation of 'specificity') is not appropriate in studies regarding sentinel node in cancer because the presence of isolated macrometastases in the sentinel node confers node positivity on the patient. Nor, given the experimental nature of sentinel node biopsy and the biological uncertainty of the significance of micrometastases, is this term appropriate for use when micrometastases alone are present in the sentinel node. Instead the term upstaging is used to better reflect the standing of sentinel nodes that are immunohistochemically positive when other non-sentinel nodes are clear of disease.

The following definitions were therefore used to ascribe the performance rates of sentinel node biopsy

Detection rate - refers to the number of times a sentinel node was actually identifiable $=$ (Number of successful attempts to retrieve a sentinel node/Number of attempts to retrieve a sentinel node) $* 100 \%$.

Accuracy rate refers to the ability of the sentinel node to reflect the overall status of the lymph basin (whether positive or negative $)=[$ (Number of correct predictions of the nodal status by sentinel node biopsy/Number of patients undergoing sentinel node biopsy)*100\%].

Sensitivity refers to the number of times the sentinel node reflects the fact that disease is present in the non-sentinel nodes $=($ Number of patients with tumor-involved sentinel nodes/Number of patients with any lymph node containing tumor) $* 100 \%$.

The false negative rate reflects the proportion of patients in whom no cancer was identified in the sentinel node but who had nodal deposits found in their non-sentinel nodes compared to the total number of those who had tumour containing metastases in non-sentinel nodes = (Number of false negative patients/Number of true positive cases + number of false-negative cases) $* 100 \%$.

Upstaging rate refers to the number of cases in which sophisticated analysis of the sentinel node reveals tumor deposits that otherwise would not have been detected $=$ (Number of patients revealing micrometastases or isolated tumor cells in the sentinel node/Number of patients 
classified as NO after routine histopathological examination) $* 100 \%$.

\section{Results}

\section{Results of literature search}

There were no randomized controlled trails identified by our search methodology. Sixty-three clinical studies regarding lymphatic mapping and sentinel node biopsy for colon cancer in human patients were published in the English language during our review period of interest. Nine of these studies however actually utilized a primarily ex vivo lymph node identification technique (only the dye injection was performed in vivo and the surgeon made no attempt intraoperatively to identify any mapped nodes). These studies [28-37] (along with ten additional studies that utilized a primary ex vivo technique ab initio [3846]) were therefore excluded from further analysis (see Figure 1 and Table 2). A further two studies were explicitly declared updates or further analyses of already reported patient cohorts.
This left fifty-two studies warranting consideration for inclusion in this study [21,47-97](see Table 3). Although ten of these studies supplemented their in vivo work with additional ex vivo examination on a occasional basis (particularly in cases of failed mappings or rectal cancers), these were included in the review as their majority of cases underwent their mapping and biopsy entirely in vivo prior to specimen resection. In addition, aside from any contribution to multicenter trials, seven centers provided 27 studies to the literature base. Eight publications have come from the John Wayne Cancer Institute alone and seven have come from the McLaren Regional Center, Michigan State University alone (with a further four publications emerging from these two centers jointly). Two centers (Wake Forest, North Carolina and MD Anderson, Texas) have published two studies each while three centers (Mount Sinai, Miami Beach; Charité-University Medicine Berlin, Germany and the University Medical Center Groningen, Netherlands) have published two studies each. However, although some degree of overlap between patient cohorts is likely in successive publications that exact proportion of patients presented in duplicate was

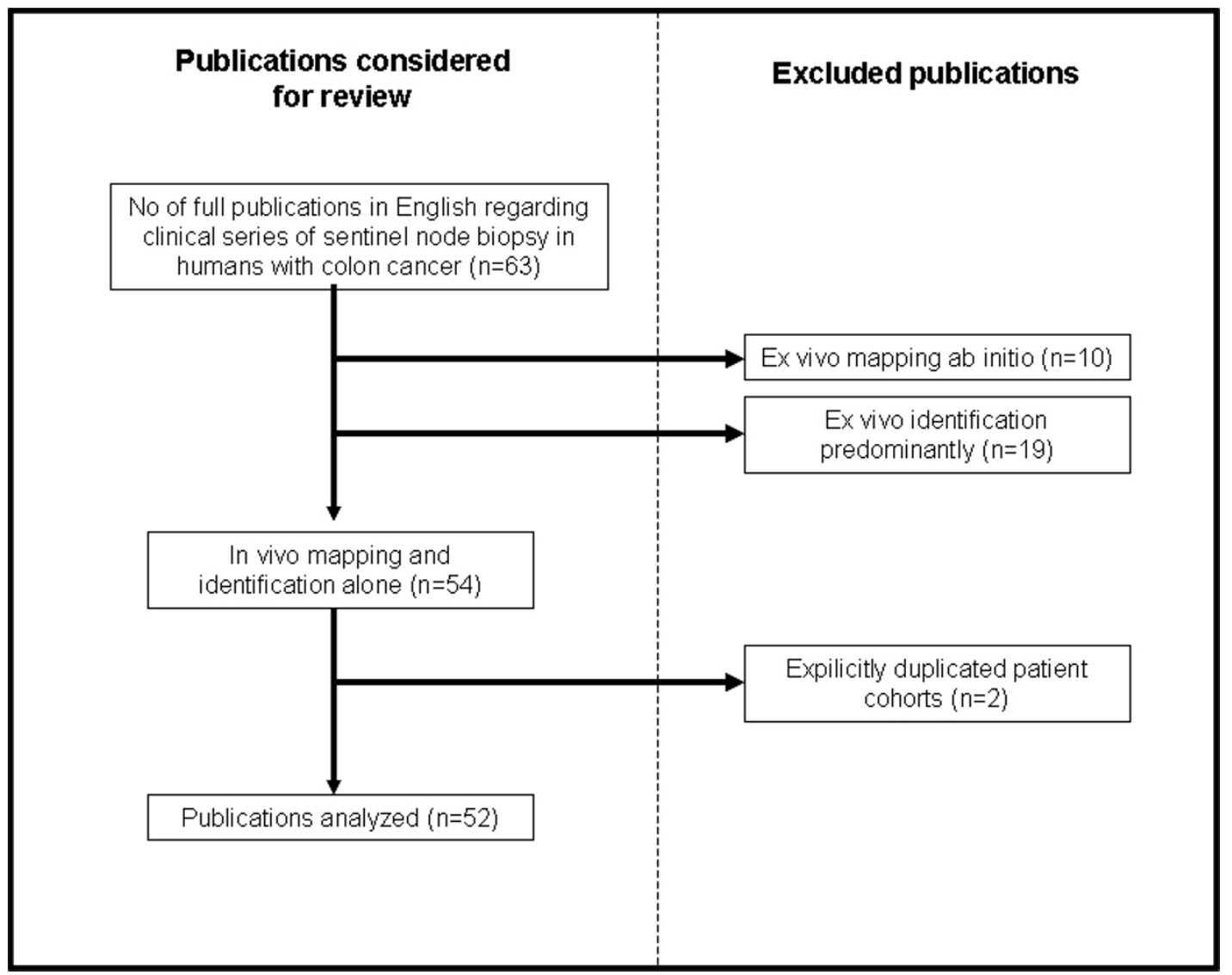

Figure I

Flow chart showing the selection and exclusion of publications for this review. 
Table 2: Studies excluded from analysis as primarily reports of ex vivo biopsy techniques.

\begin{tabular}{|c|c|c|c|c|c|}
\hline \multicolumn{3}{|c|}{ Entirely ex vivo technique } & \multicolumn{3}{|c|}{ In vivo injection but ex vivo identification of mapped nodes } \\
\hline Lead Author & Year & Journal & Lead Author & Year & Journal \\
\hline Wong [28] & 2001 & Ann Surg & Cserni [38] & 1999 & Path Oncol Res \\
\hline Fitzgerald [29] & 2002 & J Surg Oncol & Joosten [39] & 1999 & $\mathrm{Br} J$ Surg \\
\hline Broderick-Villa [30] & 2004 & Am Surg & Merrie [40] & 2001 & Dis Colon Rectum \\
\hline Wong $[31]$ & 2004 & Ann Surg Oncol & Gandy [4I] & 2002 & Colorectal Dis \\
\hline Smith F [32] & 2005 & Ann Surg Oncol & Evangelista [42] & 2002 & Tumori \\
\hline Bell [33] & 2005 & Dis Col Rectum & Demirbas [43] & 2004 & Turk J Gastroenterol \\
\hline Smith J [34] & 2006 & Am J Surg & Krishnan [44] & 2005 & Indian J Gastroenterol \\
\hline Yagik [35] & 2007 & Int J Col Dis & Van Scheltinga [45] & 2006 & Scand J Gastroenterol \\
\hline Van Schaik [36] & 2007 & Eur J Surg Oncol & Faerden AE [46] & 2008 & Dis Colon Rectum \\
\hline Stojadinovic [37] & 2007 & Ann Surg & & & \\
\hline
\end{tabular}

not explicitly stated and so all studies were included albeit with flagging.

\section{Included studies}

In total fifty two diagnostic studies (listed in Table 3) detailing 3390 patient episodes were included in this analysis. Only two studies (comprising a total of 41 patients) $[52,91]$ however set out specifically to evaluate lymphatic mapping selectively for early stage disease. This obviously is not a sufficient cohort to draw robust conclusions about the performance of the technique in this cohort. The clinical and methodological diversity (compounded by variations in data presentation) of the remaining forty-eight studies however was such that formal meta-analysis to allow statistical analysis supplement the power was neither possible nor prudent (QUADROS data not shown). Therefore this systematic review of the literature on this subject must take the form of a narrative synthesis of the evidence base in its entirety[98].

Sentinel node detection rates and false negative scores are listed alongside each publication in Table 3 and displayed graphically in Figure 2. As can be seen considerable variation in both detection rates (58-100\%) and, especially, in false negative rates $(0-75 \%)$ is evident. To be of any clinical utility the technique would seem to require these rates to be consistently better than $90 \%$ and $10 \%$ respectively (i.e. similar to that deemed acceptable for clinical use of the technique in breast cancer). Forty-five studies present identification rates greater than this threshold (indeed 34 had rates greater than $95 \%$ while twelve identified sentinel nodes in $100 \%$ of their patients). Twenty studies reported a false negative rate of $10 \%$ or below (although eight of these studies have been published from the two centers with the most publications) and eight studies reported a false negative rate of less than $5 \%$. While these data suggest that the technique could be apposite for the determination of operative extent, the eight and thirty studies $(16 \%$ and $60 \%$ respectively of all the published experience) that fail to demonstrate such levels of efficacy raise considerable concern over its reliability and reproducibility. However the same factors that preclude metaanalysis (i.e. the considerable heterogeneity regarding variable patient cohorts and tumor characteristics as well as disparate surgeon experience and protocol differences in both the technical performance and pathological analysis) may also underlie these discordant results and will now therefore be critically analyzed in sequence. Tables 4 , 5 and 6 detail the salient findings of each study by each of these criteria.

\section{Patient Demographics (Table 4)}

Ten studies gave no indication of mean (or median) patient age while eleven gave no breakdown of the patient population by gender. The age breakdown of the other studies show no especially striking data (mean age 69 years) although three studies have an unexplained clear preponderance $(>70 \%)$ of males as have two of females among their cohorts. This suggests that their populations may be somewhat atypical. Only four studies present data regarding the BMIs of their patient population - a potential important discrepancy that may induce error in both detection and false negative rates as intra-abdominal obesity may obscure discolored nodes in the mesentery. Finally, only $25(50 \%)$ studies examined colon cancer in isolation. The vast majority (26) of the other studies also included rectal cancer. These studies, despite usually declaring the proportions of each tumor studied, most often did not present result sub-analysis. While the mean number of colon cancers studied in each publication is 68,30 studies included less than 50 of such patients while 14 comprised less than 30 patients with colon cancer.

\section{Tumor profiles (Table 5)}

Despite the stated aim of most studies being the evaluation of the utility of lymphatic mapping for staging node negative tumors, 19 studies made no attempt to exclude patients with distant metastases or indeed evident 
Table 3: Publications included for this analysis along with their concluding results regarding the rates of detection, accuracy, sensitivity, false negative and upstaging as well as the false negative rates associated with lymphatic mapping and sentinel node biopsy for colon cancer.

\begin{tabular}{|c|c|c|c|c|c|c|c|c|}
\hline Lead Author & Year & Journal & $\begin{array}{l}\text { Detection } \\
\text { Rate }\end{array}$ & $\begin{array}{l}\text { Accuracy } \\
\text { Rate }\end{array}$ & $\begin{array}{l}\text { Sensitivity } \\
\text { Rate }\end{array}$ & $\begin{array}{c}\text { False Negative } \\
\text { Rate }\end{array}$ & $\begin{array}{c}\text { Negative } \\
\text { Predictive } \\
\text { Value }\end{array}$ & $\begin{array}{c}\text { Upstaging } \\
\text { Rate }\end{array}$ \\
\hline Saha (a)[47] & 2000 & Ann Surg Oncol & 99 & 96 & 91 & 9 & Ns & 18 \\
\hline Wiese (a) [48] & 2000 & Arch Pathol Lab Med & 99 & 96 & 91 & 9 & ns & ns \\
\hline Waters (b)[49] & 2000 & Am Surg & 91 & 100 & 100 & 0 & 100 & 6 \\
\hline Bilchik (c) [50] & 2001 & J Clin Oncol & 100 & 100 & 100 & 0 & 100 & 50 \\
\hline Paramo (d) $[5 I]$ & 2001 & Am J Surg & 71 & 100 & 100 & 0 & 100 & 11 \\
\hline Wood (a)+(c)[52] & 2001 & Ann Surg Oncol & 96 & 95 & 88 & 12 & ns & 25 \\
\hline Wood (c)[53] & 2001 & Surg Endosc & 100 & 100 & 100 & 0 & 100 & 9 \\
\hline Saha $(a)+(c)[54]$ & 2001 & Ann Surg Oncol & 98 & 96 & 90 & 10 & 95 & 14 \\
\hline Esser [55] & 2001 & Dis Colon Rectum & 58 & 94 & 67 & 33 & 94 & 7 \\
\hline Bendavid [56] & 2002 & J Surg Oncol & 90 & 94 & 95 & 5 & 91 & 42 \\
\hline Paramo (d)[57] & 2002 & Ann Surg Oncol & 82 & 98 & 93 & 7 & 97 & 11 \\
\hline Wood (c)[58] & 2002 & J Gastrolnt Surg & 97 & 95 & 92 & 8 & ns & 24 \\
\hline Bilchik (c)[59] & 2002 & Eur J Cancer & 97 & 95 & 91 & 5 & ns & 24 \\
\hline Kitagawa [60] & 2002 & Dis Colon Rectum & 91 & 92 & 82 & 18 & 88 & ns \\
\hline Feig (e)[6I] & 2002 & Am J Surg & 98 & 79 & 38 & 62 & 76 & 8 \\
\hline Broderick-Villa [62] & 2002 & Cancer J & 92 & 79 & 50 & 50 & 73 & 0 \\
\hline Tsioulias (c)[63] & 2002 & Am Surg & 100 & 93 & 67 & 33 & ns & 15 \\
\hline Nastro [64] & 2002 & Tumori & 75 & 100 & 100 & 0 & 100 & ns \\
\hline Bilchik (c)[65] & 2003 & Cancer Control & 100 & 93 & 91 & 9 & ns & 14 \\
\hline $\operatorname{Cox}[66]$ & 2003 & Curr Surg & 100 & 100 & 100 & 0 & 100 & 29 \\
\hline Bilchik (c)[67] & 2003 & J Clin Oncol & 96 & 96 & 92 & 8 & ns & 29 \\
\hline Turner (c)[68] & 2003 & Archives Path & 82 & 92 & 87 & 13 & ns & 29 \\
\hline Trocha (a)+(c)[69] & 2003 & J Gastrolnt Surg & 98 & 95 & 84 & 16 & 93 & 21 \\
\hline Veihl [70] & 2003 & World J Surg & 87 & 78 & 50 & 22 & 71 & 11 \\
\hline Levine (b)[7I] & 2003 & J Gastrolnt Surg & 92 & ns & 50 & ns & 86 & ns \\
\hline Saha (a)[72] & 2004 & Dis Colon Rectum & 99 & ns & 88 & 12 & ns & 13 \\
\hline $\operatorname{Dan}(\mathrm{a})[73]$ & 2004 & Arch Surg & 99 & 96 & 86 & 16 & ns & 5 \\
\hline Braat $[2 I]$ & 2004 & Eur J Surg Oncol & 94 & 97 & 80 & 20 & 92 & 3 \\
\hline Bertoglio [74] & 2004 & J Surg Oncol & 95 & 92 & 78 & 22 & 88 & ns \\
\hline Read [75] & 2004 & Dis Colon Rectum & 79 & 97 & 25 & 75 & ns & 3 \\
\hline Patten (e)[76] & 2004 & Cancer & 98 & 89 & 83 & 17 & 76 & 20 \\
\hline Bertagnolli [77] & 2004 & Ann Surg & 92 & 80 & 46 & 54 & 75 & 0 \\
\hline Saha (a) [78] & 2004 & Ann Surg Oncol & 100 & 95 & 84 & 16 & 93 & 5 \\
\hline Saha (a) [79] & 2004 & Semin Oncol & 100 & 96 & 92 & 8 & ns & 13 \\
\hline Bembenek (f) [80] & 2005 & World J Surg & 85 & ns & 92 & 8 & 95 & 26 \\
\hline Codnignola [8I] & 2005 & JJ Clin Oncol & 100 & ns & 72 & 28 & 70 & 37 \\
\hline Dahl [82] & 2005 & Eur J Surg Oncol & 100 & 92 & 83 & 17 & 91 & ns \\
\hline Bilchik (a)+(c)[83] & 2006 & Arch Surg & 100 & 95 & 88 & 12 & ns & 23 \\
\hline Tuech [84] & 2006 & Eur J Surg Oncol & 97 & 94 & 91 & 9 & ns & ns \\
\hline Saha (a)[85] & 2006 & Am J Surg & 98 & 96 & 90 & 10 & 93 & ns \\
\hline Kelder $(\mathrm{g})[86]$ & 2006 & Scand J Gastroenterol & 97 & 93 & 86 & 14 & 89 & 33 \\
\hline Thomas (b)[87] & 2006 & Am Surg & 93 & 20 & 46 & 54 & 73 & 5 \\
\hline Covarelli [88] & 2006 & Am Surg & 95 & 95 & 86 & 14 & ns & 8 \\
\hline Kelder $(\mathrm{g})[89]$ & 2007 & Int J Col Dis & 97 & 96 & 89 & 11 & 93 & ns \\
\hline Bianchi [90] & 2007 & Surg End & 100 & 95 & 83 & 17 & 94 & 9 \\
\hline Murawa [91] & 2007 & Acta Chir Belg & 93 & 84 & 83 & 17 & ns & 8 \\
\hline Bembenek (f)[92] & 2007 & Ann Surg & 85 & 86 & 54 & 46 & 80 & 21 \\
\hline Sandrucci [93] & 2007 & J Surg Oncol & 100 & 91 & 92 & 9 & ns & 11 \\
\hline Tiffet [94] & 2007 & Dis Colon Rectum & 92 & 81 & 80 & 20 & 74 & ns \\
\hline $\operatorname{Lim}(e)[95]$ & 2008 & Ann Surg Oncol & 99 & 83 & 59 & 41 & 78 & ns \\
\hline Kusano [96] & 2008 & Digestive Surgery & 88.5 & 82.6 & 33 & 67 & 81 & ns \\
\hline Quadros [97] & 2008 & J Surg Oncol & 91 & 80 & 67 & 33 & 67 & 25 \\
\hline
\end{tabular}

The definitions for each are contained in the text. All rates are \%; ns denotes data neither stated within nor readily derived from the results presented within the publication. Note: several centers have published more than one series without declaring overlap of their patient cohorts such publications are flagged by the center of origin by the inclusion of letter subscript as follows: (a) McLaren Regional Center, Michigan, USA; (b) Wake Forest, North Carolina, USA; (c) John Wayne Cancer Institute, California, USA; (d) Mount Sinai, Miami Beach, USA; (e) MD Anderson, Texas; (f) Charité-University Medicine Berlin, Berlin, Germany; (g) University Medical Center Groningen, The Netherlands. Table 4,5 and 6 also follow a similar format. 
mesenteric deposits let alone grossly involved lymph nodes. Indeed some actually specifically included such patients. Of the remainder, seven excluded only patients with distant metastases (making no further consideration of local tumour invasion or mesenteric deposits) while nine required the patient had only 'clinically localized' or 'resectable' disease to allow their inclusion. Of the twelve papers that exclude gross nodal and distant metastases, five include T4 disease while five do not profile their tumors by T-stage. Overall, at least 21 studies include T4 tumors within their cohorts. Furthermore, 25 of the studies that present such data possess high T3 and T4 to T1 and T2 ratios. Only five studies specifically consider tumor length or diameter as a factor that may affect performance parameters. Interestingly, four of these studies conclude that false negative rates are considerably more likely in patients with larger tumors while the fifth only examined this by taking $4 \mathrm{~cm}$ as a cut-off point for analysis. Only one study considered how tumor size may relate to the quantity of dye needed to adequately map it and found a significant positive correlation. Although the mean number of resected nodes in each study often is adequate (mean overall is in 15.3), 21 studies include patients who have had considerably less nodes than this resected in their 'definitive' operation while 25 do not state either the mean or range of the non-sentinel nodal harvest. This raises concern over the quality control mechanisms in place to ensure the standard of the oncological operation performed in these studies.

\section{Technical methodology (Table 6)}

Only five studies specifically sought to ensure surgeon experience in the technique prior to commencing their study (although an additional nine had already demonstrated their competence in a prior publication). This is likely particularly pertinent in the ten multicentre studies, only four of whom specifically sought to ensure minimum practical experience among their participants (although a further three studies came from units that had already published experiences and so this likely evinces expertise at least among some of the contributors). Injection methodology overall was relatively similar. 45 protocols utilized an intraoperative subserosal injection of colorimetric mapping agent while three employed a submucosal injection. Twenty eight studies utilized isosulfan blue $1 \%$ in isolation while ten used Patent Blue alone and one used Vital Blue. Eight studies used a radioisotope as a mapping agent (alone in one study and in conjunction with blue dye in the others) and the majority injected this agent submucosally preoperatively by additional endoscopy. Two studies also incorporated fluorescein while one used indocyanine green. Six studies specifically included laparoscopic operations with three employing this approach exclusively. All commenting authors agreed however that the technique is easily performed regardless of operative approach and adds minimally to overall operative time[63]. The mean number of sentinel nodes found was consistently approximately two although five studies included within their range numbers in excess of double this. There was though considerable variation among how identified nodes were histologically analyzed. Four studies examined only a single section of the sentinel node while nine used neither immunohistochemistry nor RT-PCR to look for micrometastases or isolated tumor cells (one of these did however subsequently publish an additional, detailed report on their results with such techniques[99]).

Despite the fact that the variation in accuracy and sensitivity rates is frequently decried, only fifteen publications specifically included analysis of their false negative rates (see Table 7 for a tabulated summary of their conclusions). Twelve of these found that increasing tumor stage was inversely related to non-sentinel node tumors and indeed in five studies the detection rate and diagnostic accuracy was $100 \%$ among their T1 and T2 cohorts. One other study found that the presence of lymphovascular invasion was significantly associated with false negative rates but that lymph node invasion did not reach significance as a predictor (no data was shown however). The remaining study analyzing its results by tumor stage found no significance difference with either tumor stage or an arbitrarily decided lesion diameter.

\section{Critical analysis of studies with low performance results}

The nine studies with detection rates $<90 \%$ and the thirtytwo studies with false negative rates $>10 \%$ were then scrutinized from the perspectives gleaned from these analyses. Interestingly, five of the nine studies $(>50 \%)$ with low detection rates also had false negative rates greater than $20 \%$ (actually 22\%, 33\%, 46\% and 75\%). Of the 43 studies with detection rates $>90 \%$, only eleven (c. $25 \%$ ) also had false negative rates greater than $20 \%$.

Of those with detection rates $<90 \%$, two were multicentric trials. Neither these nor six of the seven single centre studies stated they validated surgeon expertise prior to commencing patient enrollment. Furthermore each of these studies was composed of less than 60 patients. Five studies included a proportion of rectal cancers approximating $15 \%$ of the population within their study cohorts. Four studies had marked T3/4 to T1/2 preponderance (in the order of $93: 3,78: 22$ and 83:15 respectively). Only three studies of those with detection rates $>90 \%$ included such high proportions of locally advanced disease but one of these specifically excluded clinically apparent lymphadenopathy while the other did not contain any T4 cases. Furthermore one other study included patients with liver metastases and even obvious mesenteric deposits and direct nodal invasion by the primary while every 
Table 4: Cohort characteristics with regard to baseline demographics of the patients studied.

\begin{tabular}{|c|c|c|c|c|c|c|}
\hline Lead Author & $\%$ Males & Mean or median age & $\begin{array}{c}\text { BMI } \\
\left(\mathbf{k g} / \mathrm{m}^{2}\right)\end{array}$ & $\begin{array}{c}\text { Non-colonic cancers } \\
\text { included }\end{array}$ & $\%$ with colon cancer & No with colon cancer \\
\hline Saha & 46 & 71 & ns & Rectum & 86 & 74 \\
\hline Wiese & 44 & 71 & ns & Rectum & 70 & 70 \\
\hline Waters & ns & ns & ns & None & 100 & 22 \\
\hline Bilchik & 43 & 70 & ns & Rectum & 83 & 33 \\
\hline Paramo & 46 & 72 & ns & None & 100 & 35 \\
\hline Wood & 40 & 68 & ns & Rectum & 81 & 61 \\
\hline Wood & 36 & 64 & ns & None & 100 & 11 \\
\hline Saha & ns & ns & ns & Rectum & ns & ns \\
\hline Esser & 55 & 69 & ns & Rectum & 84 & 26 \\
\hline Bendavid & ns & ns & ns & None & 100 & 20 \\
\hline Paramo & 51 & 70 & ns & None & 100 & 55 \\
\hline Wood & 49 & 68 & ns & Rectum & 78 & 78 \\
\hline Bilchik & 49 & 68 & ns & Rectum & 100 & 100 \\
\hline Kitagawa & 71 & 61 & ns & Rectum & 21 & 12 \\
\hline Feig & 73 & 68 & ns & None & 100 & 48 \\
\hline Broderick-Villa & 50 & 63 & ns & Rectum & 90 & 40 \\
\hline Tsioulias & ns & ns & ns & None & 100 & 14 \\
\hline Nastro & ns & ns & ns & None & 100 & 8 \\
\hline Bilchik & 12 & 64 & ns & None & 100 & 30 \\
\hline Cox & 35 & Ns & ns & None & 100 & 17 \\
\hline Bilchik & 48 & 70 & ns & Rectum & 85 & 102 \\
\hline Turner & 53 & 76 & ns & Rectum & 86 & 44 \\
\hline Trocha & 56 & 71 & ns & Rectum & 88 & 44 \\
\hline Veihl & 74 & 75 & 25.3 & None & 100 & 31 \\
\hline Levine & 55 & 67 & ns & Stomach & 74 & 37 \\
\hline Saha & 48 & 71 & ns & Rectum & 83 & 336 \\
\hline Dan & 49 & 72 & ns & Rectum & 88 & 106 \\
\hline Braat & ns & ns & ns & None & 100 & 35 \\
\hline Bertoglio & 54 & ns & ns & Rectum & 77 & 20 \\
\hline Read & ns & ns & ns & None & 100 & 41 \\
\hline Patten & ns & 64.2 & ns & None & 100 & 50 \\
\hline Bertagnolli & 65 & 65 & ns & None & 100 & 72 \\
\hline Saha & 47 & 71 & ns & Rectum & 91 & 52 \\
\hline Saha & 48 & 71.3 & ns & Rectum & 80 & 209 \\
\hline Bembenek & 49 & 45 to 83 & ns & None & 100 & 55 \\
\hline Codnignola & 36 & 70.8 & ns & Rectum & 93 & 52 \\
\hline Dahl & 57 & 70 & ns & None & 100 & 30 \\
\hline Bilchik & 47 & 74 & ns & Rectum & 73 & 97 \\
\hline Tuech & ns & 75.5 & ns & None & 100 & 30 \\
\hline Saha & 48 & 74 & ns & Rectum & 82 & 408 \\
\hline Kelder & 53 & 69 & ns & None & 100 & 30 \\
\hline Thomas & 50 & 67 & c. 26.3 & None & 100 & 69 \\
\hline Covarelli & 60 & 70 & ns & None & 100 & 20 \\
\hline Kelder & ns & ns & ns & None & 100 & 69 \\
\hline Bianchi & 58 & 61 & ns & None & 100 & 22 \\
\hline Murawa & ns & 61 & ns & Rectum & 48 & 13 \\
\hline Bembenek & 59 & 67 & ns & None & 100 & 315 \\
\hline Sandrucci & ns & 73 & ns & Rectum & 86 & 30 \\
\hline Tiffet & 50 & 73 & 25 & Rectum & 75 & 49 \\
\hline Lim & 48 & 67 & ns & None & 100 & 120 \\
\hline Kusano & 70 & 70 & ns & None & 100 & 26 \\
\hline Quadros & 36.5 & 56 & ns & Rectum & 42 & 22 \\
\hline
\end{tabular}

The order and format is the same as in Table 3.

patient in another study was conventionally node positive. Finally the patients of one report had a mean BMI above $25 \mathrm{~kg} / \mathrm{m}^{2}$ (although this was not analyzed specifi- cally further). None of the other studies presented any data in this latter regard. 
Table 5: Patient selection criteria along with the resulting tumor profiles in each of the studies and the mean total lymph node harvests.

\begin{tabular}{|c|c|c|c|c|c|c|c|c|c|c|}
\hline \multirow[t]{2}{*}{ Lead Author } & \multirow[t]{2}{*}{$\begin{array}{l}\text { Selection criteria } \\
\text { employed }\end{array}$} & \multirow[t]{2}{*}{$\begin{array}{c}\text { Tumor Size } \\
\text { (cm) }\end{array}$} & \multirow[b]{2}{*}{ Tis } & \multicolumn{4}{|c|}{$\begin{array}{c}\text { T Stage } \\
\text { (\% of total) }\end{array}$} & \multirow[t]{2}{*}{ TI 2:34 ratio } & \multirow{2}{*}{$\begin{array}{c}\text { Conventionally } \\
\text { Node Negative } \\
(\%)\end{array}$} & \multirow{2}{*}{$\begin{array}{l}\text { No of lymph } \\
\text { nodes resected } \\
\text { (range) }\end{array}$} \\
\hline & & & & TI & $\mathbf{T} 2$ & T3 & T4 & & & \\
\hline Saha & None & ns & & \multicolumn{4}{|c|}{ Data not given } & ns & 37 & 16 (ns) \\
\hline Wiese & None & ns & 0 & 14 & 22 & 53 & 11 & $36: 63$ & 41 & 16 (ns) \\
\hline Waters & None & ns & \multicolumn{5}{|c|}{ Data not given } & ns & 27 & 12 (ns) \\
\hline Bilchik & $\begin{array}{l}\text { Early stage primary } \\
\text { only }\end{array}$ & ns & 0 & 26 & 24 & \multicolumn{2}{|c|}{50} & $50: 50$ & 35 & $15(2-28)$ \\
\hline Paramo & $\begin{array}{c}\text { No distant metastatic } \\
\text { disease }\end{array}$ & ns & 0 & 12 & 7 & 81 & 0 & 7:93 & 29 & 10 (ns) \\
\hline Wood & $\begin{array}{c}\text { Clinically localized } \\
\text { primary }\end{array}$ & ns & 0 & 19 & 29 & 44 & 8 & $48: 52$ & 47 & $15(2-28)$ \\
\hline Wood & $\begin{array}{l}\text { Small early stage } \\
\text { cancers only }\end{array}$ & 1.4 & 27 & 54 & 9 & 9 & 0 & $91: 9$ & 9 & $13(2-20)$ \\
\hline Saha & None & ns & \multicolumn{5}{|c|}{ Data not given } & ns & 40 & 20 (ns) \\
\hline Esser & $\begin{array}{l}\text { No nodal or distant } \\
\text { metastases }\end{array}$ & ns & \multicolumn{5}{|c|}{ Data not given } & ns & 19.4 & $15(12-16)$ \\
\hline Bendavid & None & ns & \multicolumn{5}{|c|}{ Data not given } & ns & 65 & ns \\
\hline Paramo & $\begin{array}{c}\text { No distant metastatic } \\
\text { disease }\end{array}$ & ns & \multicolumn{5}{|c|}{ Data not given } & ns & 27 & 12 (ns) \\
\hline Wood & Clinically localized & ns & \multicolumn{5}{|c|}{ Data not given } & ns & 26 & $15(2-28)$ \\
\hline Bilchik & Clinically localized & ns & 0 & 25 & 23 & 46 & 6 & $48: 52$ & 43 & $15(3-28)$ \\
\hline Kitagawa & $\begin{array}{l}\text { Only if curative } \\
\text { surgery }\end{array}$ & ns & \multicolumn{5}{|c|}{ Data not given } & $29: 71$ & 43 & 24 (ns) \\
\hline Feig & None & ns & 7 & 6 & 23 & 58 & 6 & $36: 64$ & 33 & $13(4-46)$ \\
\hline Broderick-Villa & $\begin{array}{l}\text { No known distant } \\
\text { metastases }\end{array}$ & $?$ & 6 & 8 & 20 & 62 & 4 & $34: 66$ & 43 & $8(1-17)$ \\
\hline Tsioulias & $\begin{array}{c}\text { Clinically localized } \\
\text { only }\end{array}$ & ns & \multicolumn{5}{|c|}{ Data not given } & ns & 21 & $14(2-2 \mid)$ \\
\hline Nastro & None & ns & \multicolumn{5}{|c|}{ Data not given } & ns & ns & ns \\
\hline Bilchik & $\begin{array}{c}\text { Early stage primary } \\
\text { only }\end{array}$ & ns & 20 & 46 & 14 & 20 & 0 & $80: 20$ & 21 & $14(2-2 \mid)$ \\
\hline Cox & None & ns & 0 & 6 & 36 & 58 & 0 & $42: 58$ & 41 & $18(4-33)$ \\
\hline Bilchik & $\begin{array}{c}\text { Early stage primary } \\
\text { only }\end{array}$ & 3.6 & 14 & 12 & 17 & 53 & 5 & $42: 58$ & 36 & 14 (ns) \\
\hline Turner & None & ns & 0 & 12 & 10 & 75 & 4 & $22: 78$ & 52 & II (I-42) \\
\hline Trocha & $\begin{array}{l}\text { No distant } \\
\text { metastases }\end{array}$ & ns & 26 & 12 & 18 & 42 & 2 & $56: 44$ & 38 & 16 (ns) \\
\hline Veihl & None & 4.2 & 0 & 6 & 9 & 71 & 12 & $15: 83$ & 48 & $21(5-40)$ \\
\hline Levine & $\begin{array}{l}\text { No gross nodal } \\
\text { disease }\end{array}$ & ns & \multicolumn{5}{|c|}{ Data not given } & ns & ns & ns \\
\hline Saha & $\begin{array}{c}\text { Tumor resectable \& } \\
\text { no metastases }\end{array}$ & ns & \multicolumn{5}{|c|}{ Data not given } & ns & 42 & ns \\
\hline
\end{tabular}


Table 5: Patient selection criteria along with the resulting tumor profiles in each of the studies and the mean total lymph node harvests. (Continued)

\begin{tabular}{|c|c|c|c|c|c|c|c|c|c|c|}
\hline Dan & None & ns & 17 & 15 & 13 & 53 & 3 & ns & 43 & ns \\
\hline Braat & $\begin{array}{c}\text { No distant } \\
\text { metastases, gross } \\
\text { invasion or nodal } \\
\text { disease }\end{array}$ & ns & 0 & 6 & 20 & 51 & 23 & $26: 74$ & 34 & $9(1-23)$ \\
\hline Bertoglio & $\begin{array}{l}\text { Stage I and II \& no } \\
\text { enlarged nodes only }\end{array}$ & ns & & \multicolumn{4}{|c|}{ Data not given } & ns & 65 & $13(6-18)$ \\
\hline Read & $\begin{array}{l}\text { Surgery with curative } \\
\text { intent only }\end{array}$ & ns & & \multicolumn{4}{|c|}{ Data not given } & ns & 29 & $14(7-45)$ \\
\hline Patten & $\begin{array}{l}\text { No nodal or distant } \\
\text { metastases }\end{array}$ & ns & & \multicolumn{4}{|c|}{ Data not given } & $37: 63$ & 39 & 14 (ns) \\
\hline Bertagnolli & Stage I, II and III only & ns & 0 & 29 & 16 & 46 & 9 & $35: 65$ & 67 & 17 (ns) \\
\hline Saha & None & ns & 19 & 14 & 11 & 53 & 4 & $43: 57$ & 33 & 12 (ns) \\
\hline Saha & None & ns & 11 & 10 & 15 & 51 & 5 & $34: 66$ & 41 & 14 (ns) \\
\hline Bembenek & $\begin{array}{l}\text { Conventionally node } \\
\text { negative patients only }\end{array}$ & ns & \multicolumn{5}{|c|}{ Data not given } & ns & 100 & $26(10-59)$ \\
\hline Codnignola & No liver metastases & ns & 0 & 2 & 21 & 63 & 14 & $23: 77$ & 36 & $21(6-47)$ \\
\hline Dahl & $\begin{array}{c}\text { No nodal or distant } \\
\text { metastases }\end{array}$ & ns & \multicolumn{5}{|c|}{ Data not given } & $11: 88$ & 40 & $17(4-35)$ \\
\hline Bilchik & $\begin{array}{l}\text { Potentially curable } \\
\text { cancer with no } \\
\text { distant metastases } \\
\text { only }\end{array}$ & $3.5(0.2-10.5)$ & 0 & 17 & 15 & 65 & 3 & $32: 68$ & 29 & 15 (ns) \\
\hline Tuech & $\begin{array}{c}\text { No nodal or distant } \\
\text { disease }\end{array}$ & ns & 0 & 6 & 9 & 85 & 0 & $10: 90$ & 36 & $20(12-32)$ \\
\hline Saha & None & ns & 15 & 11 & 16 & 52 & 5 & $42: 58$ & 50 & 15 (ns) \\
\hline Kelder & $\begin{array}{l}\text { No gross nodal or } \\
\text { distant metastases }\end{array}$ & ns & 0 & 0 & 23 & 73 & 4 & $23: 77$ & 21 & 14 (ns) \\
\hline Thomas & None & ns & \multicolumn{5}{|c|}{ Data not given } & $?$ & 38 & ns \\
\hline Covarelli & None & ns & \multicolumn{5}{|c|}{ Data not given } & ns & 35 & ns \\
\hline Kelder & $\begin{array}{l}\text { No gross nodal or } \\
\text { distant metastases }\end{array}$ & ns & 0 & 1 & 20 & 70 & 9 & $21: 79$ & $4 I$ & II (ns) \\
\hline Bianchi & $\begin{array}{c}\text { No T4 or metastatic } \\
\text { disease }\end{array}$ & ns & 36 & 4.5 & 9 & 45 & 5 & $50: 50$ & 73 & $22(8-38)$ \\
\hline Murawa & $\begin{array}{l}\text { No gross nodal or } \\
\text { distant metastases }\end{array}$ & ns & 0 & 15 & 20 & 63 & 2 & $37: 63$ & 41 & $20(3-96)$ \\
\hline Bembenek & None & ns & \multicolumn{5}{|c|}{ Data not given } & ns & 69 & $20(4-79)$ \\
\hline Sandrucci & Stage I or II only & ns & \multicolumn{5}{|c|}{ Data not given } & $100: 0$ & 69 & 9 (ns) \\
\hline Tiffet & $\begin{array}{l}\text { Excluded if primary } \\
\text { unresectable. }\end{array}$ & ns & \multicolumn{5}{|c|}{ Data not given } & $22: 78$ & 41 & $18(4-37)$ \\
\hline Lim & $\begin{array}{l}\text { No gross nodal or } \\
\text { distant metastases }\end{array}$ & ns & 0 & 4 & 26 & 66 & 4 & $30: 70$ & 16 & 13(ns) \\
\hline Kusano & None & ns & 0 & 15 & 73 & \multicolumn{2}{|c|}{12} & $88: 12$ & 77 & 13.5 (ns) \\
\hline Quadros & $\begin{array}{l}\text { Potential curable } \\
\text { cancer, no distant } \\
\text { metastases }\end{array}$ & 8.3 & 0 & 0 & 14 & 54 & 31 & $14: 86$ & 46 & 19 (ns) \\
\hline
\end{tabular}

The order and format is the same as in Table 3. 
Table 6: Surgeon and technical factors associated with nodal mapping, identification and pathological analysis methodology.

\begin{tabular}{|c|c|c|c|c|c|c|c|c|}
\hline \multirow[b]{2}{*}{ Lead Author } & \multirow[b]{2}{*}{$\begin{array}{l}\text { Surgeon } \\
\text { Experience } \\
\text { sought }\end{array}$} & \multirow[b]{2}{*}{ Multicentre } & \multirow[b]{2}{*}{$\begin{array}{l}\text { Laparoscopic } \\
\text { resection (\%) }\end{array}$} & \multirow[b]{2}{*}{$\begin{array}{c}\text { Mapping agent } \\
\text { used }\end{array}$} & \multirow[b]{2}{*}{$\begin{array}{c}\text { Supplementay } \\
\text { ex vivo } \\
\text { identification }\end{array}$} & \multirow[b]{2}{*}{$\begin{array}{l}\text { Mean/median } \\
\text { No. of sentinel } \\
\text { nodes (range) }\end{array}$} & \multicolumn{2}{|c|}{ Sentinel node analysis } \\
\hline & & & & & & & $\begin{array}{c}\text { Serial } \\
\text { sectioning }\end{array}$ & $\begin{array}{l}\text { Immunohist. } \\
\text { or RT-PCR }\end{array}$ \\
\hline Saha & No & No & ns & Isosulfan blue I\% & No & $1.6(\mathrm{I}$ to 4$)$ & Yes & No \\
\hline Wiese & No & No & 0 & Isosulfan blue I\% & No & $1.9(1$ to 4$)$ & Yes & Yes \\
\hline Waters & No & No & ns & Isosulfan blue I\% & No & ns & Yes & Yes \\
\hline Bilchik & Yes & Yes & ns & Isosulfan blue I\% & No & $2(1$ to 3$)$ & Yes & Yes \\
\hline Paramo & No & No & 0 & Isosulfan blue I\% & No & $1.4(1$ to 4$)$ & Yes & Yes \\
\hline Wood & No & No & 12 & Isosulfan blue I\% & Yes $(10 \%)$ & $2(1$ to 4$)$ & Yes & Yes \\
\hline Wood & No & No & 100 & Isosulfan blue I\% & No & $2(1$ to 3$)$ & Yes & Yes \\
\hline Saha & No & Yes & ns & Isosulfan blue I\% & No & $1.7(1$ to 4$)$ & Yes & Yes \\
\hline Esser & No & No & 0 & Isosulfan blue I\% & No & $1.7(0$ to 5$)$ & No & No \\
\hline Bendavid & No & No & ns & Isosulfan blue I\% & No & $3.9(0$ to 5$)$ & Yes & Yes \\
\hline Paramo & No & No & 0 & Isosulfan blue I\% & No & $1.6(0$ to 4$)$ & Yes & Yes \\
\hline Wood & No & No & ns & Isosulfan blue I\% & Yes (15\%) & $2(\mid$ to 4$)$ & Yes & Yes \\
\hline Bilchik & No & No & 16 & Isosulfan blue I\% & Yes (11\%) & $2(1$ to 4$)$ & Yes & Yes \\
\hline Kitagawa & No & No & 0 & Techneticum & No & $3.5(0$ to 8$)$ & No & No \\
\hline Feig & No & Yes & 0 & Isosulfan blue I\% & No & $2.6(0$ to 7$)$ & No & Yes \\
\hline Broderick-Villa & No & No & 0 & Isosulfan blue I\% & Yes (23\%) & $1.5(0$ to 5$)$ & Yes & Yes \\
\hline Tsioulias & No & No & 100 & Isosulfan blue I\% & No & $1.7(1$ to 3$)$ & Yes & Yes \\
\hline Nastro & No & No & 0 & $\begin{array}{l}\text { Technetium and } \\
\text { blue dye }\end{array}$ & No & ns & Yes & Yes \\
\hline Bilchik & Yes & No & 23 & Isosulfan blue I\% & No & $2(1$ to 3$)$ & Yes & Yes \\
\hline Cox & No & No & 0 & Isosulfan blue I\% & Yes $(58 \%)$ & $6(2$ to II) & Yes & Yes \\
\hline Bilchik & Yes & No & ns & Isosulfan blue I\% & No & 1.75 (ns) & Yes & Yes \\
\hline Turner & Yes & No & ns & Isosulfan blue I\% & No & $3(\mathrm{~ns})$ & Yes & Yes \\
\hline Trocha & No & No & 0 & $\begin{array}{l}\text { Technetium and } \\
\text { blue dye }\end{array}$ & No & $2.5(\mathrm{~ns})$ & Yes & Yes \\
\hline Veihl & No & Yes & 0 & Isosulfan blue I\% & No & $2(1$ to 8$)$ & Yes & Yes \\
\hline Levine & No & No & 0 & Isosulfan blue I\% & No & $1.9(1$ to 6$)$ & No & Yes \\
\hline Saha & Yes & No & ns & Isosulfan blue I\% & No & 2.1 (ns) & Yes & Yes \\
\hline Dan & No & No & 0 & $\begin{array}{l}\text { Isosulfan and } \\
\text { fluorescein }\end{array}$ & No & $2.5(\mathrm{~ns})$ & Yes & Yes \\
\hline Braat & No & No & ns & Patent Blue & Yes (57\%) & $1.7(\mathrm{I}$ to 4$)$ & Yes & Yes \\
\hline Bertoglio & No & No & ns & Vital Blue & No & $2.9(1$ to 3$)$ & Yes & No \\
\hline Read & No & No & 0 & Isosulfan blue I\% & No & $2(\mathrm{~ns})$ & No & No \\
\hline Patten & No & No & 0 & $\begin{array}{l}\text { Technetium and } \\
\text { patent blue }\end{array}$ & No & $3.5(0$ to $\mathrm{II})$ & Yes & Yes \\
\hline Bertagnolli & No & Yes & 0 & Isosulfan blue I\% & No & 2.1 (ns) & Yes & No \\
\hline Saha & No & No & 0 & $\begin{array}{l}\text { Technetium and } \\
\text { Isosulfan blue }\end{array}$ & No & $3(1$ to 4$)$ & Yes & Yes \\
\hline Saha & No & Yes & 0 & $\begin{array}{l}\text { Isosulfan, } \\
\text { Technetium and } \\
\text { fluorescein }\end{array}$ & No & $2(1$ to 4$)$ & Yes & Yes \\
\hline Bembenek & No & No & 0 & Patent Blue & No & $2(\mathrm{~ns})$ & Yes & Yes \\
\hline Codnignola & No & No & 0 & Patent Blue & No & 2.02 (ns) & Yes & Yes \\
\hline Dahl & No & No & 0 & $\begin{array}{l}\text { Patent Blue } \\
\text { (with } \\
\text { radioisotope in } \\
\text { six) }\end{array}$ & Yes (6\%) & $2.2(0$ to 6$)$ & Yes & No \\
\hline Bilchik & Yes & Yes & 15 & Isosulfan blue I\% & Yes (4\%) & 3 (ns) & Yes & Yes \\
\hline Tuech & Yes & No & ns & Patent Blue & Yes & $1.5(\mathrm{~ns})$ & Yes & Yes \\
\hline Saha & Yes & Yes & ns & Isosulfan blue I\% & No & $2.2(\mathrm{~ns})$ & Yes & yes \\
\hline Kelder & No & No & 0 & Patent Blue & No & 2.7 (I to 4$)$ & Yes & Yes \\
\hline Thomas & No & No & 0 & Isosulfan blue I\% & No & 2.1 (I to 5$)$ & Yes & yes \\
\hline Covarelli & No & No & 0 & $\begin{array}{l}\text { Technetium and } \\
\text { blue dye }\end{array}$ & No & $\mathrm{I} .3$ (ns) & Yes & Yes \\
\hline Kelder & Yes & Yes & 0 & Patent Blue & No & $2,3(\mathrm{~ns})$ & Yes & Yes \\
\hline Bianchi & No & No & 100 & Patent Blue & Yes (5\%) & $2(\mathrm{~ns})$ & Yes & Yes \\
\hline Murawa & No & No & 0 & Patent Blue & No & $1.6(0$ to 4$)$ & No & Yes \\
\hline Bembenek & No & Yes & 7 & Patent Blue & No & ns & Yes & Yes \\
\hline Sandrucci & No & No & ns & $\begin{array}{l}\text { Patent blue and } \\
\text { technetium }\end{array}$ & No & $2.2(\mathrm{~ns})$ & Yes & No \\
\hline Tiffet & No & No & 0 & $\begin{array}{l}\text { Patent blue and } \\
\text { technetium }\end{array}$ & No & $2.6(n s)$ & Yes & Yes \\
\hline Lim & No & No & ns & $\begin{array}{l}\text { Technetium and } \\
\text { Isosulfan blue }\end{array}$ & Yes & $4(\mathrm{~ns})$ & Yes & Yes \\
\hline Kusano & No & No & $62 \%$ & $\begin{array}{l}\text { Indocyanine } \\
\text { green }\end{array}$ & No & $2.6(0-5)$ & No & No \\
\hline Quadros & No & No & 0 & $\begin{array}{l}\text { Technetium and } \\
\text { patent blue }\end{array}$ & Yes & $3.5(\mathrm{~ns})$ & Yes & Yes \\
\hline
\end{tabular}

The order and format is the same as in Table 3. 
Of the sixteen studies with false negative rates above $20 \%$, twelve presented no critical analysis of their false negative rates. Nor did any of these studies place any emphasis on surgeon experience in their stated inclusion criteria. Four studies were performed on a multicentre basis but none explicitly ensured surgeon expertise prior to commencement (in one such study the mean number of operations per surgeon was less than three) and nine studies included non-colonic tumors in between 10 and $26 \%$ of the cohort size. Furthermore one study specifically commented that tumors adherent to the retroperitoneum were included while five had T3/4 tumors accounting for approximately $80 \%$ of their patient cohorts. Three had a mean tumor size of greater than $4.2 \mathrm{~cm}$ with one concluding that its false negatives case were associated with significantly bigger tumor sizes $(7.2 \mathrm{~cm}$ in the false negative cases versus 5.2 $\mathrm{cm}$ mean size overall). One additional study also found a strong trend in favor of an association between false negative rate and larger tumor sizes $(4.5 \mathrm{~cm} \mathrm{v} 3.5 \mathrm{~cm}, \mathrm{p} \mathrm{=}$ 0.09 ). Two studies had a mean number of resected lymph nodes of eight and nine respectively while at least twelve studies included some patients with less than ten nodes in their resected specimens (eight including some with five or less nodes examined). Two others presented no mean data on this subject and three presented no range. Four did not serially section the sentinel node while six did not employ immunohistochemistry or RT-PCR.

With respect to the fifteen publications with false negative rates between 10 and $20 \%$, only three studies included specific analysis of their rates. Of the fifteen, two were multicentric trials and both sought to ensure surgical expertise and had false negative rates each of 11 and $12 \%$. Nine studies included rectal cancers in their cohorts (in between $9 \%$ and $74 \%$ of their cohorts). Four studies had no explicit exclusion criteria while six sought only to exclude distant disease deposits. No study provided any data on patient BMI. Only one study specifically excluded T4 disease. Eight had a significant $(>60 \%)$ T3/4 preponderance (being $>70 \%$ ). The mean number of resected lymph nodes was greater than ten in fourteen. Four studies included patients with less than this number while the one presented no data regarding the range. Two studies did not employ serial sectioning of the identified sentinel nodes and two did not utilize immunohistochemical or RT-PCR methods of examination.

\section{Discussion}

Sufficient lymph basin resection is an oncologic sine non qua of operation with curative intent for solid organ malignancies as nodal status remains the primary portent of prognosis and adjuvant therapy prescription. For colon cancer, staging propriety by convention demands that this equate to en bloc resection of the entire mesenteric lymphatic delta. While the manner of performance of stand- ard resectional operation for colon cancer by laparoscopy or laparotomy means that the extent of access is already determined (and so supplementing bowel resection with full mesenteric resection is readily facilitated) this is not for case for endoscopic resectional techniques. Therefore these techniques are currently limited to the address of benign lesions or neoplastic disease with minimum likelihood of lymphatic dissemination. A minimally invasive means of reliably confirming the lymph node status could greatly enhance the oncological propriety of these approaches and extend their indications towards their actual technical capacity. While it is clear that sentinel node biopsy is not indicated to minimize the extent of therapeutic lymphadenectomy in colon cancer (i.e. the resection of nodes containing tumor deposits), it could theoretically have a role in helping select those with truly early stage disease for endoscopic resection.

Furthermore, although it is often considered that adjoining mesenteric lymph node dissection to the intestinal resection impacts little on the patient undergoing conventional oncological operation for colon cancer [100-103], this assumption does not necessarily stand up to close scrutiny. The high-tie of the arterial inflow arcade necessary to adequately perform mesenteric nodal dissection however segues wider longitudinal intestinal margins than would otherwise be necessary for local clearance purposes alone. The negative impact of wide dissection and resection may include post-operative bowel dysfunction, nerve plexus praxia or adjacent organ injury [104-106]. Therefore, tacit knowledge, as well as some prior clinical investigation [107-109], would suggest that minimizing the extent of mesenteric dissection could pay dividends for the patient even if standard surgical access is employed. These considerations have however been lost amid the concerns regarding the efficacy of lymphatic mapping in this disease and the current emphasis on ensuring adequate staging by resection of considerable 'minimum' numbers of lymph nodes [110-112].

The focus of sentinel node biopsy in colon cancer has therefore been on upstaging conventionally node negative patients after conventional radical operation[113]. This means that false-negative sentinel nodes do not impose any negative effect on the patient but also has conferred a priori license to investigators to modify the technique and broaden the patient cohort. To propose that it could be used a means of predicting a negative basin and obviating mesenteric dissection places great emphasis on ensuring that the technique's rationale in colon cancer is biologically sound and that methodological discrepancies are eliminated. However the literature base to date contains multiple un-standardized variables and so assessment of the true utility of lymphatic mapping when applied selectively to early stage disease is considerably confounded. 

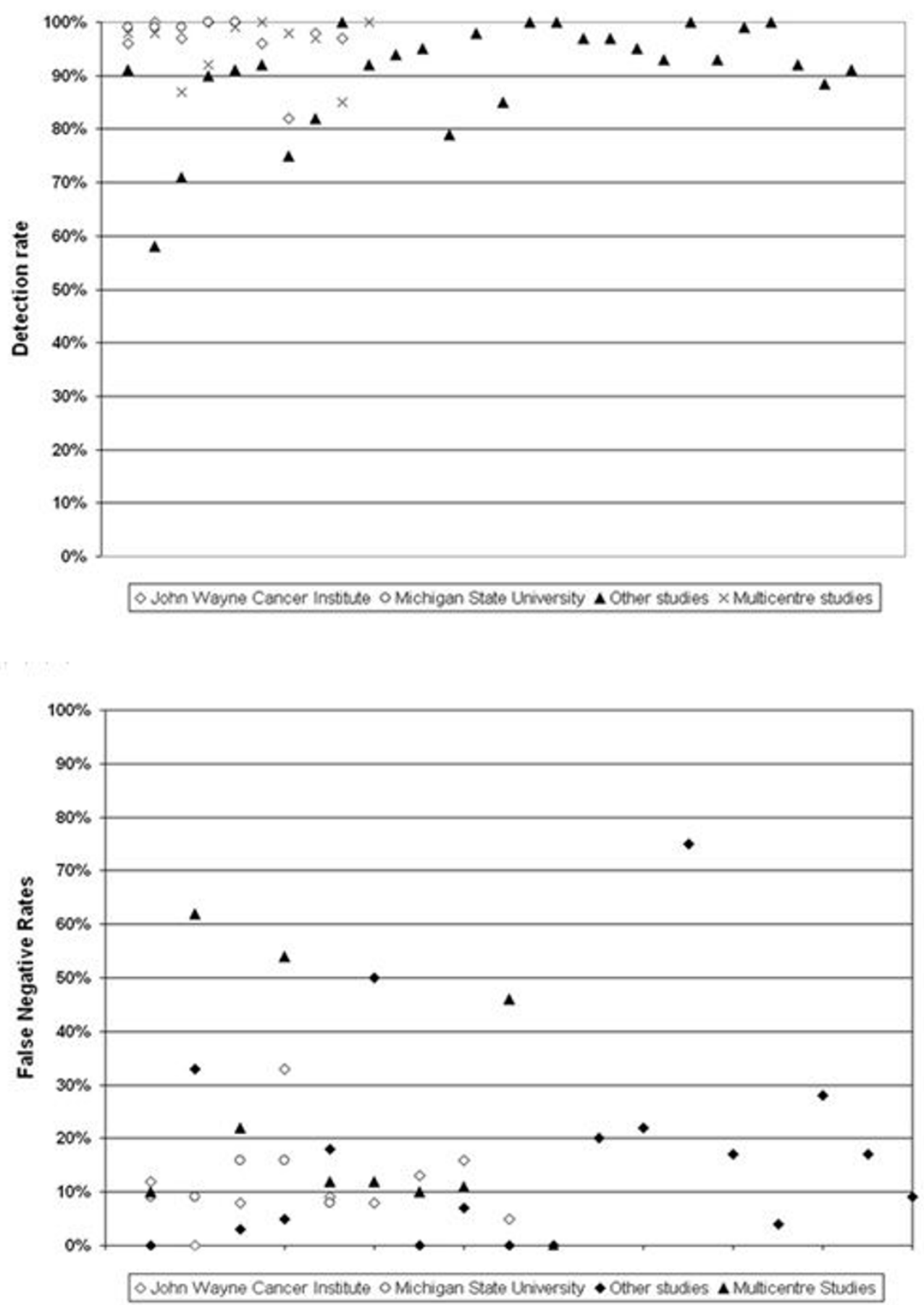

\section{Figure 2}

Spread of (a) Detection rates and (b) False Negative Rates among the studies of in vivo sentinel node mapping in colon cancer included in this review. In the figures, studies arising from either of the two centers with the most publications (ie The John Wayne Cancer Institute, California and the McLaren Reginal Cancer Centre, Michgan) on the topic are indicated separately to minimize any visual bias resulting from their inclusion. The remaining studies are divided into multicenter studies and all others. 
Table 7: Tabulated summary of the specific analyses of failed or false negative analysis where such has been explicitly contained within the publication.

\begin{tabular}{|c|c|c|}
\hline Authors & Year & Comment \\
\hline Bendavid [56] & 2002 & $\begin{array}{l}\text { The one false negative case occurred in a patient with liver metastases. } \\
\text { Also 'evidently metastatic nodes' did not receive colourant }\end{array}$ \\
\hline Paramo [57] & 2002 & No specific analysis presented. \\
\hline Wood [58] & 2002 & $\begin{array}{l}\text { All five false negatives occurred in T3 or T4 tumors (in one case the only positive non-sentinel node was involved by } \\
\text { direct extension). Three occurred in Ist } 30 \text { cases }\end{array}$ \\
\hline Bilchik [59] & 2002 & All five false negatives occurred in T3 or T4 tumors. Three occurred in the first fifty cases. \\
\hline Kitagawa [60] & 2002 & Four false negative cases were advanced $\mathrm{T} 3$ and/or had massive lymph node metastases \\
\hline Feig [6I] & 2002 & Also 'several patients' (of ten) classified as false negative had 'palpable lymph nodes' \\
\hline Broderick-Villa [62] & 2002 & $\begin{array}{l}\text { Learning curve strongly associated with false negative rate }(67 \% \text { in first half, } 32 \% \text { in second half). No significant } \\
\text { association with T-stage, } L N \text { involvement or tumor diameter }>\text { or }<4 \mathrm{~cm}\end{array}$ \\
\hline Veihl [70] & 2003 & $\begin{array}{l}\text { Amount of dye relative to tumor size was an important predictor of identification of node. False negative more } \\
\text { common in cases with larger nodes }(4.5 \mathrm{~cm} v 3.4 \mathrm{~cm}, \mathrm{p}=0.09)\end{array}$ \\
\hline Bilchik [83] & 2006 & Of the six false negatives, four were attributable to tumor obliteration of the lymphatic channels \\
\hline Saha $[85]$ & 2006 & $95 \%$ of patients with skip metastases were $\mathrm{T} 3$ or $\mathrm{T} 4$ \\
\hline Thomas [87] & 2006 & $\begin{array}{l}\text { Two patients with liver metastases along with two others with gross mesenteric disease had false positive sentinel } \\
\text { nodes. No relationship between BMI and disease }\end{array}$ \\
\hline Kelder [89] & 2006 & In one of the two false negatives, the non-SLNs were involved by extra-nodal tumor invasion \\
\hline Bembenek [92] & 2007 & $\begin{array}{l}\text { Significant association with learning curve/center experience, BMI (cut-off level being } 22 \text { patients and a BMI of } 25 \\
\text { respectively) \& LVI. No significant association between detection and T stage, age, sex, vascular invasion, no of nodes, } \\
\text { total no of nodes. }\end{array}$ \\
\hline Sandrucci [93] & 2007 & 'Skip metastases' were all correlated with 'T2 lesions with massive lymphatic involvement' \\
\hline Tiffet [94] & 2007 & $\begin{array}{l}\text { Three of } 12 \text { false negatives were in patients with direct tumor involvement of adjacent non-sentinel epicolic nodes } \\
\text { while four were in N2 patients. False negative rate markedly lower in the subgroup with } \mathrm{TI} \text { and } \mathrm{T} 2 \text { tumors only. and } \\
\text { in those with } \mathrm{BMI}<30 \mathrm{~kg} / \mathrm{m}^{2}\end{array}$ \\
\hline
\end{tabular}

The high accuracy rates in some studies do at least suggest that its concept is biologically tenable (indeed of those centers that have published multiple series, four conclude strongly in favor of the reliability of the technique) and that methodological variability may underlie the discordant results (as they do for the same technique in malignancies at different sites). However, this heterogeneity also confounds any attempt at meta-analysis to selectively extract data regarding the reliability of sentinel node biopsy selectively in early stage cancer. A similar conclusion was also reached by another group performing a detailed statistical systematic review of the literature from another perspective[114]. Although their review contains significant differences in study inclusion and exclusion criteria to ours, it is interesting that they nonetheless concluded that the technique had a $9 \%$ median risk of a false negative result in comparison to an $8.4 \%$ median risk for the technique in breast cancer. However, the general tendency to pool results in the reporting of studies frustrated their attempt to relate T-stage to sensitivity.

The first evident confounder to obtaining clarity regarding the accuracy of the technique is the number of publications emanating from single centers. Although it seems likely that these seven centers (who together have contributed over half of the published evidence base) have overlapped their patient cohorts at least to some extent in successive publications, the exact proportions is rarely explicitly declared. Equally however it cannot be assumed that these studies entirely overlapped their experiences and so to ensure fairness and transparency every study meeting the inclusion exclusion is included in this study with those coming from the same center being flagged in the Tables. The next main obstacle within each publication complicating deliberation of the technique's applicability for early stage colon cancer is the marked contamination of rectal cancers throughout the evidence base. The consequences of doing so without presentation of complete subgroup analyses gives an artificial impression of reduced feasibility and accuracy rates overall because lymphatic mapping is clearly more arduous and less reliable in this site[115]. Furthermore, specific account of tumor size and mural penetration in colon cancer is too often lacking for any firm conclusions to be made regarding the efficacy of selectively mapping early stage disease. The main inclusion criterion to date is primarily the requirement for operation for symptomatic colon cancer despite the fact that the disease tends to present late (often at stage when curative surgery is not possible and adjuvant treatment is already indicated regardless of the nodal status). An elongated diameter of the cancer may also mean that different segments of the tumor map to different nodes as watershed areas are crossed. Furthermore, direct spread through the bowel wall may compress and alter the original lymphatic flow and so cause the mapping agent to deviate away from the 
original first order draining nodes. Extensive nodal involvement, including total destruction of the nodes by metastases, may also compromise the identification of true sentinel nodes[22,116]. Such concerns have been confirmed in the analyses presented here as well as in ex vivo experiences of mapping colon cancer and indeed in other tumors [117-119].

Surgeon expertise and experience has already been determined a major feature for lymphatic mapping in breast cancer[120] and seems likely to also be one for colonic mapping (a point made clear in one study attempting ex vivo biopsy that found many of their failed mapping were associated with intraluminal dye injection)[39]. The relationship between low detection rate and higher false negative rate is a likely related phenomenon while lack of technical proficiency may in addition compound any technical difficulties associated with adverse patient factors (such as BMI or previous laparotomy) to further undermine the performance of the technique. A specific learning curve can also certainly be expected if the surgeon looks for the nodes intraoperatively. However the lack of specific inclusion criteria for this seems to suggest that many of the studies published to date are in fact reflections of expertise acquirement rather than reports of technique reliability. The timing of the vital blue dye injection prior to resection may also be a crucial issue. A prolonged latency in identifying the node (depending on the intraoperative situation) may allow overflowing of the dye and potentially complete decoloration of the first stained node. Also the discrepancy in standardizing nodal analysis between the reports is concerning as standard assessment based on hematoxylin and eosin staining of one level of a paraffin-embedded block reportedly can miss as many as 33\% of metastases[121]. While micrometastatic disease may of itself be of considerable clinical importance [115], these cells in sentinel nodes may also confer significance by their prediction of the presence of nonsentinel nodal metastases. Finally the numbers within the nodal harvest rates of many studies raises serious concerns over quality assurance utilized given that this aspect is of utmost importance when a novel technique is compared to established practice.

Certain groups have however clearly managed to overcome confounding factors of the technique and consistently obtain negative predictive values of similar quality to those that currently justify conservative resection fields in other specialties. It is perhaps no surprise that these investigators tend to fastidiously analysis their false negative results as did the pioneers of the technique in breast cancer and melanoma. On the other hand, other authorities have not hesitated to declare the technique in colon cancer either invalid or of dubious clinical value $[40,122]$ on the basis of their own experience and perception of the evidence base. Most of the latter group did not however present data detailing their efforts to examine the potential biological reasons behind their results. As much therefore as it is clear that certain proponents in both single centre and multicentre trials can perform sentinel node biopsy with high accuracy for colonic malignancy, it is equally clear that others cannot. The fact that advanced endoscopic resection is at present only wrought in supraspecialized centers may mean however that only such selected, expert departments need attempt to investigate, validate and standardize the performance of sentinel node biopsy to accompany it. Also, the need for high detection and accuracy rates implicit if the results were going to impinge on patient care could drive efforts to develop methods to improve the yield of lymphatic mapping by use of additional or alternative dyes (perhaps fluorescent markers[73,123] or radioisotopes[69,78]) as have been used for other cancers [124-126].

The general tendency of a relationship between advancing stage an increased likelihood of metastases being present in non-sentinel nodes also supports the basic contention that lymphatic mapping in colon cancer may be particularly efficacious in germinal cancers - a perspective made particularly compelling by the inherent suitability of early stage disease for truly minimally invasive resective techniques. The lesions that could be resected by endoscopic means are by definition smaller and therefore likely confined to a single lymphatic delta. Furthermore T3 or T4 disease identifiable by staging is not feasibly resected endoscopically and so the tumor stages with the highest frequency of being node-positive are excluded. The fact that $20-40 \%$ of patients with T3/4 lesions but without demonstrable lymph node metastases actually die of their disease also supports exclusion of these patients from non-radical operation. De facto confinement of the patient cohort to T1 and T2 stage disease (perhaps by including adjunctive staging measures such as endoscopic ultrasound[127]) may therefore obviate some of the main confounders of lymphatic mapping and potentially allow proffering of localized tumor resection as definitive surgery. However the lack of specific study or discriminating subgroup analysis by T-stage means that this aspect of lymphatic mapping in colon cancer has yet to be definitively explored and so this remains speculative.

If sentinel node biopsy is ever to be used as means to alleviate mesenteric resection in cases that are truly node negative, consideration must be given to the cases where the sentinel node is positive or indeed falsely negative. In the former case, subsequent radical lymphadenectomy should still be performed. Ideally therefore the sentinel node analysis should be performed intraoperatively to allow direct progress to the definitive excisional surgery (whether of the primary alone or of the traditional 
extended operation). Considerable precedent exists for such analysis in breast cancer [128-131] and it is even possible that additional innovative technology may allow for in situ virtual biopsy of the salient nodes[132,133]. In the situation that the sentinel node is falsely negative, suspicious features present in the resected specimen may encourage revisional surgery in some cases. Although reintervention after a previous laparotomy may be difficult, the risk of added morbidity because of a prior minimally invasive 'cherry-picking' of sentinel nodes is likely to be less. However any true consideration of the risk involved needs balancing with an accurate measure of the potential benefit of avoiding mesenteric resection. The consequence of missed positive lymph nodes is mainly one of potential understaging and hence depriving the patient of systemic chemotherapy. However it should also be realized the added benefit of chemotherapy over standard surgery for $\mathrm{T} 1$ and $\mathrm{T} 2$ lesions is low and perhaps that the clinical significance of minimal nodal disease in these patients is lower than that of more advanced T-stage (as is the case for early gastric cancer[134])[135,136]. The actual clinical risk of detriment due to mesenteric lymphadenopathy per se is also low[85]. It is worth however here emphasizing again that these deliberations only apply to colon cancer and not its counterpart in the rectum. The anatomic characteristics of the mesorectum (namely its bulk, extraperitoneal situation and lack of a serosal layer) mitigate against selective sentinel node biopsy in isolation for rectal cancer however as does the fact that transgression of its planes may complicate any subsequent total mesorectal resection in cases of nodal positivity and so impact upon the potential for curative resection. Finally the consequences of missed nodal disease in the pelvis and, therefore the occurrence of pelvic recurrence, may be catastrophic for the patient.

\section{Conclusion}

Sentinel node mapping could never substitute for a properly performed oncologic colorectal resection when this is indicated. The concept however that lymphatic mapping may have sufficient capability to provide the oncological proprietary for the curative surgery for early stage cancers without en bloc mesenteric resection seems biologically plausible but cannot yet be definitively judged given the lack of clarity and consistency in the literature to date. Specific study of the technique in those early stage tumors likely to be selected for endoscopic resection is clearly therefore essential before this approach can be considered in clinical practice.

\section{Competing interests}

The authors declare that they have no competing interests.

\section{Authors' contributions}

RAC conceived of the study, participated in the study design and performance (performed the initial literature review and analysis) and composed the manuscript. JL contributed to the study design and performance and guided the manuscript composition. JM conceived of the study and participated in its design and coordination. All authors read and approved the final manuscript.

\section{Acknowledgements}

RAC very gratefully acknowledges the financial grant supplied by the Royal College of Surgeons in Ireland which facilitated his participation in this study by contributing to his fellowship funding at the IRCAD/EITS. This funding body however had no role in the study design, data collection, analysis and interpretation or manuscript composition.

\section{References}

I. Tamegai Y, Saito Y, Masaki N, Hinohara C, Oshima T, Kogure E, Liu $Y$, Uemura N, Saito K: Endoscopic submucosal dissection: a safe technique for colorectal tumors. Endoscopy 2007, 39:4I 8-422.

2. Hurlstone DP, Atkinson R, Sanders DS, Thomson M, Cross SS, Brown $\mathrm{S}$ : Achieving $\mathrm{RO}$ resection in the colorectum using endoscopic submucosal dissection. Br J Surg 2007, 94: I 536-I542.

3. Adachi Y, Yasuda K, Kakisako K, Sato K, Shiraishi N, Kitano S: Histopathologic criteria for local excision of colorectal cancer: multivariate analysis. Ann Surg Oncol 1999, 6:385-388.

4. Tanaka S, Oka S, Kaneko I, Hirata M, Mouri R, Kanao H, Yoshida S, Chayama K: Endoscopic submucosal dissection for colorectal neoplasia: possibility of standardization. Gastrointest Endosc 2007, 66:100-107.

5. Mönig SP, Baldus SE, Zirbes TK, Schröder W, Lindemann DG, Dienes $\mathrm{HP}$, Hölscher AH: Lymph node size and metastatic infiltration in colon cancer. Ann Surg Oncol 1999, 6:579-58I.

6. Herrera-Ornelas L, Justiniano J, Castillo N, Petrelli NJ, Stulc JP, Mittelman A: Metastases in small lymph nodes from colon cancer. Arch Surg 1987, I 22: I 253-1256.

7. Herrera L, Villarreal JR: Incidence of metastases from rectal adenocarcinoma in small lymph nodes detected by a clearing technique. Dis Colon Rectum 1992, 35:783-788.

8. Rodriguez-Bigas MA, Maamoun S, Weber TK, Penetrante RB, Blumenson LE, Petrelli NJ: Clinical significance of colorectal cancer: metastases in lymph nodes $<\mathbf{5} \mathbf{~ m m}$ in size. Ann Surg Oncol 1996, 3:124-130.

9. Haboubi NY, Abdalla SA, Amini S, Clark P, Dougal M, Dube A, Schofield $P$ : The novel combination of fat clearance and immunohistochemistry improves prediction of the outcome of patients with colorectal carcinomas: a preliminary study. Int J Colorectal Dis 1998, I3:99-102.

10. Chok KS, Law WL: Prognostic factors affecting survival and recurrence of patients with $\mathrm{pT} I$ and $\mathrm{PT} 2$ colorectal cancer. World J Surg 2007, 3 I : | 485- | 490.

II. Kitagawa Y, Kitajima M: Endoscopic treatment combined with laparoscopic sentinel node mapping for superficial gastrointestinal cancers. Endoscopy 2007, 39:47I-475.

12. Saikawa $Y$, Otani $Y$, Kitagawa $Y$, Yoshida M, Wada N, Kubota T, Kumai K, Sugino Y, Mukai M, Kameyama K, Kubo A, Kitajima M: Interim results of sentinel node biopsy during laparoscopic gastrectomy: possible role in function-preserving surgery for early cancer. World J Surg 2006, 30:1962-1968.

13. Kitagawa Y, Fujii H, Kumai K, Kubota T, Otani Y, Saikawa Y, Yoshida $M$, Kubo A, Kitajima $M$ : Recent advances in sentinel node navigation for gastric cancer: a paradigm shift of surgical management. J Surg Oncol 2005, 90:|47-I5I.

14. Franklin ME Jr, Leyva-Alvizo A, Abrego-Medina D, Glass JL, Treviño J, Arellano PP, Portillo G: Laparoscopically monitored colonoscopic polypectomy: an established form of endoluminal therapy for colorectal polyps. Surg Endosc 2007, 2 I: I650- I653.

15. Cahill RA, Perretta S, Leroy J, Dallemagne B, Marescaux J: Lymphatic Mapping and Sentinel Node Biopsy in the Colonic Mesentery by Natural Orifice Transluminal Endoscopic Surgery (NOTES). Ann Surg Oncol 2008, I 5:2677-2683. 
16. Tuech J], Pessaux P, Regenet N, Bergamaschi R, Colson A: Sentinel lymph node mapping in colon cancer. Surg Endosc 2004, 18:1721-1729.

17. Prabhudesai AG, Kumar D: The sentinel lymph node in colorectal cancer - of clinical value? Colorectal Dis 2002, 4:162-166.

18. Mulsow J, Winter DC, O'Keane JC, O'Connell PR: Sentinel lymph node mapping in colorectal cancer. Br J Surg 2003, 90:659-667.

19. Cserni G: Nodal staging of colorectal carcinomas and sentinel nodes. J Clin Pathol 2003, 56:327-335.

20. de Haas RJ, Wicherts DA, Hobbelink MG, Borel Rinkes IH, Schipper ME, Zee JA van der, van Hillegersberg R: Sentinel lymph node mapping in colon cancer: current status. Ann Surg Oncol 2007, 14:1070-1080.

21. Braat AE, Oosterhuis JW, Moll FC, de Vries JE: Successful sentinel node identification in colon carcinoma using Patent Blue V. Eur J Surg Oncol 2004, 30:633-637.

22. Doekhie FS, Peeters KC, Kuppen PJ, Mesker WE, Tanke HJ, Morreau $H$, Velde $C J$ van de, Tollenaar RA: The feasibility and reliability of sentinel node mapping in colorectal cancer. Eur J Surg Oncol 2005, $31: 854-862$

23. Bembenek A, Gretschel S, Schlag PM: Sentinel lymph node biopsy for gastrointestinal cancers. J Surg Oncol 2007, 96:342-352.

24. Iddings $D$, Bilchik $A$ : The biologic significance of micrometastatic disease and sentinel lymph node technology on colorectal cancer. J Surg Oncol 2007, 96:67I-677.

25. Bilchik $A$, Comptom C: Close collaboration between surgeon and pathologist is essential for accurate staging of early colon cancer. Ann Surg 2007, 245:864-866.

26. Whiting P, Rutjes AWS, Reitsma JB, Bossuyt PM, Kleijnen J: The development of QUADAS: a tool for the quality assessment of studies of diagnostic accuracy included in systematic reviews. BMC Med Res Methodol 2003, 3:25.

27. Westwood ME, Whiting PF, Kleijnen J: How does study quality affect the results of a diagnostic meta-analysis? BMC Med Res Methodol 2005, 5:20

28. Wong JH, Steineman S, Calderia C, Bowles J, Namiki T: Ex vivo sentinel node mapping in carcinoma of the colon and rectum. Ann Surg 200I, 233:515-52I.

29. Fitzgerald TL, Khalifa MA, Al Zahrani M, Law CH, Smith AJ: Ex vivo sentinel lymph node biopsy in colorectal cancer: a feasibility study. J Surg Oncol 2002, 80:27-32.

30. Broderick-Villa G, Amr D, Haigh PI, O'Connell TX, Danial T, Difronzo LA: Ex vivo lymphatic mapping: a technique to improve pathologic staging in colorectal cancer. Am Surg 2004, 70:937-941.

3I. Wong JH, Johnson DS, Namiki T, Tauchi-Nishi P: Validation of ex vivo lymphatic mapping in hematoxylin-eosin node-negative carcinoma of the colon and rectum. Ann Surg Oncol 2004, I I:772-777.

32. Smith FM, Coffey JC, Khasri NM, Walsh MF, Parfrey N, Gaffney E, Stephens R, Kennedy MJ, Kirwan W, Redmond HP: Sentinel nodes are identifiable in formalin-fixed specimens after surgeonperformed ex vivo sentinel lymph node mapping in colorectal cancer. Ann Surg Oncol 2005, I 2:504-509.

33. Bell SW, Mourra N, Fléjou JF, Parc R, Tiret E: Ex vivo sentinel lymph node mapping in colorectal cancer. Dis Colon Rectum 2005, 48:74-79.

34. Smith J, Hwang $\mathrm{H}$, Wiseman KW, Filipenko D, Phang PT: Ex vivo sentinel lymph node mapping in colon cancer: improving the accuracy of pathologic staging? Am J Surg 2006, 19 1:665-668.

35. Yagci G, Unlu A, Kurt B, Can MF, Kaymakcioglu N, Cetiner S, Tufan $T$, Sen D: Detection of micrometastases and skip metastases with ex vivo sentinel node mapping in carcinoma of the colon and rectum. Int / Colorectal Dis 2007, 22:167-173.

36. Van Schaik PM, Linden JC van der, Ernst MF, Gelderman WA, Bosscha $\mathrm{K}$ : Ex vivo sentinel lymph node "mapping" in colorectal cancer. Eur J Surg Oncol 2007, 33: I |77-1 | 82.

37. Stojadinovic A, Nissan A, Protic M, Adair CF, Prus D, Usaj S, Howard RS, Radovanovic D, Breberina M, Shriver CD, Grinbaum R, Nelson JM, Brown TA, Freund HR, Potter JF, Peretz T, Peoples GE: Prospective randomized study comparing sentinel lymph node evaluation with standard pathologic evaluation for the staging of colon carcinoma: results from the United States Military Cancer Institute Clinical Trials Group Study GI-0I. Ann Surg 2007, 245:846-857.
38. Cserni G, Vajda K, Tarián M, Bori R, Svébis M, Baltás B: Nodal staging of colorectal carcinomas from quantitative and qualitative aspects. Can lymphatic mapping help staging? Pathol Oncol Res 1999, 5:29|-296.

39. Joosten JJ, Strobbe LJ, Wauters CA, Pruszczynski M, Wobbes T, Ruers T): Intraoperative lymphatic mapping and the sentine node concept in colorectal carcinoma. $\mathrm{Br}$ 」 Surg 1999, 86:482-486

40. Merrie AE, van Rij AM, Phillips LV, Rossaak JI, Yun K, McCall JL: Diagnostic use of the sentinel node in colon cancer. Dis Colon Rectum 200I, 44:4I0-4I7.

4I. Gandy CP, Biddlestone LR, Roe AM, O'Leary DP: Intra-operative injection of Patent Blue $\mathbf{V}$ dye to facilitate nodal staging in colorectal cancer. Colorectal Dis 2002, 4:447-449.

42. Evangelista W, Satolli MA, Malossi A, Mussa B, Sandrucci S: Sentine lymph node mapping in colorectal cancer: a feasibility study. Tumori 2002, 88:37-40.

43. Demirbaş S, Ince M, Baloğlu H, Celenk T: Should sentinel lymph node mapping be performed for colorectal cancer? Turk J Gastroenterol 2004, 15:39-44.

44. Krishnan P, Chandrika K, Sudheer OV, Dhar P, Sudhindran S: Is sentinel node mapping useful in colorectal carcinoma? Indian J Gastroenterol 2005, 24: I 29-130.

45. Van Scheltinga SE, Den Boer FC, Pijpers R, Meyer GA, Engel AF, Silvis $R$, Meijer S, Sijp JR van der: Sentinel node staging in colon carcinoma: value of sentinel lymph node biopsy with radiocolloid and blue staining. Scand J Gastroenterol Suppl 2006, 243: I53-I 57.

46. Faerden $\mathrm{AE}$, Sjo OH, Andersen SN, Hauglann B, Nazir N, Gravdehaug B, Moberg I, Svinland A, Nesbakken A, Bakka A: Sentinel node mapping does not improve staging of lymph node metastasis in colonic cancer. Dis Colon Rectum 2008, 5 I:89I-896.

47. Saha S, Wiese D, Badin J, Beutler T, Nora D, Ganatra BK, Desai D, Kaushal S, Nagaraju M, Arora M, Singh T: Technical details of sentinel lymph node mapping in colorectal cancer and its impact on staging. Ann Surg Oncol 2000, 7:120-124.

48. Wiese DA, Saha S, Badin J, Ng PS, Gauthier J, Ahsan A, Yu L: Pathologic evaluation of sentinel lymph nodes in colorectal carcinoma. Arch Pathol Lab Med 2000, I 24:1759-1763.

49. Waters GS, Geisinger KR, Garske DD, Loggie BW, Levine EA: Sentinel lymph node mapping for carcinoma of the colon: a pilot study. Am Surg 2000, 66:943-945

50. Bilchik AJ, Saha S, Wiese D, Stonecypher JA, Wood TF, Sostrin S, Turner RR, Wang HJ, Morton DL, Hoon DS: Molecular staging of early colon cancer on the basis of sentinel node analysis: a multicenter phase II trial. J Clin Oncol 200I, 19:1 128-II36.

5I. Paramo JC, Summerall J, Wilson C, Cabral A, Willis I, Wodnicki H, Poppiti R, Mesko TW: Intraoperative sentinel lymph node mapping in patients with colon cancer. Am / Surg 200I, 182:40-43.

52. Wood TF, Saha S, Morton DL, Tsioulias G], Rangel D, Hutchinson W Jr, Foshag LJ, Bilchik AJ: Validation of lymphatic mapping in colorectal cancer: in vivo, ex vivo, and laparoscopic techniques. Ann Surg Oncol 200I, 8: I50-I57.

53. Wood TF, Spirt M, Rangel D, Shen P, Tsioulias G], Morton DL, Bilchik AJ: Lymphatic mapping improves staging during laparoscopic colectomy for cancer. Surg Endosc 200I, 15:715-719.

54. Saha S, Bilchik A, Wiese D, Espinosa M, Badin J Ganatra BK, Desai D, Kaushal S, Singh T, Arora M: Ultrastaging of colorectal cancer by sentinel lymph node mapping technique - a multicenter trial. Ann Surg Oncol 200I, 8(9 Suppl):94S-98S.

55. Esser S, Reilly WT, Riley LB, Eyvazzadeh C, Arcona S: The role of sentinel lymph node mapping in staging of colon and rectal cancer. Dis Colon Rectum 200I, 44:850-854.

56. Bendavid Y, Latulippe JF, Younan RJ, Leclerc YE, Dube S, Heyen F, Morin M, Girard R, Bastien E, Ferreira J, Cerino M, Dubé P: Phase I study on sentinel lymph node mapping in colon cancer: a preliminary report. J Surg Oncol 2002, 79:8I-84.

57. Paramo JC, Summerall J, Poppiti R, Mesko TW: Validation of sentinel node mapping in patients with colon cancer. Ann Surg Oncol 2002, 9:550-554.

58. Wood TF, Nora DT, Morton DL, Turner RR, Rangel D, Hutchinson W, Bilchik AJ: One hundred consecutive cases of sentinel lymph node mapping in early colorectal carcinoma: detection of missed micrometastases. J Gastrointest Surg 2002, 6:322-329.

59. Bilchik AJ, Nora D, Tollenaar RA, Velde CJ van de, Wood T, Turner $\mathrm{R}$, Morton DL, Hoon DS: Ultrastaging of early colon cancer 
using lymphatic mapping and molecular analysis. Eur J Cancer 2002, 38:977-985.

60. Kitagawa $\mathrm{Y}$, Watanabe $M$, Hasegawa $H$, Yamamoto $S$, Fujii $H$, Yamamoto K, Matsuda J, Mukai M, Kubo A, Kitajima M: Sentinel node mapping for colorectal cancer with radioactive tracer. Dis Colon Rectum 2002, 45: 1476-1480.

6I. Feig BW, Curley S, Lucci A, Hunt KK, Vauthey JN, Mansfield PF, Cleary K, Hamilton S, Ellis V, Brame M, Berger DH: caution regarding lymphatic mapping in patients with colon cancer. $\mathrm{Am} J$ Surg 200I, 182:707-712

62. Broderick-Villa G, Ko A, O'Connell TX, Guenther JM, Danial T, DiFronzo LA: Does tumor burden limit the accuracy of lymphatic mapping and sentinel lymph node biopsy in colorectal cancer? Cancer J 2002, 8:445-450.

63. Tsioulias GJ, Wood TF, Spirt M, Morton DL, Bilchik AJ: A novel lymphatic mapping technique to improve localization and staging of early colon cancer during laparoscopic colectomy. Am Surg 2002, 68:56I-565.

64. Nastro P, Sodo M, Dodaro CA, Gargiulo S, Acampa W, Bracale U, Renda A: Intraoperative radiochromoguided mapping of sentinel lymph node in colon cancer. Tumori 2002, 88:352-353.

65. Bilchik AJ, Trocha SD: Lymphatic mapping and sentinel node analysis to optimize laparoscopic resection and staging of colorectal cancer: an update. Cancer Control 2003, 10:219-223.

66. Cox ED, Kellicut D, Adair C, Marley K, Otchy DP, Peoples GE: Sentinel lymph node evaluation is technically feasible and may improve staging in colorectal cancer. Curr Surg 2002, 59:30I-306.

67. Bilchik AJ, Nora DT, Sobin LH, Turner RR, Trocha S, Krasne D, Morton DL: Effect of lymphatic mapping on the new tumor-nodemetastasis classification for colorectal cancer. J Clin Oncol 2003, $21: 668-672$

68. Turner RR, Nora DT, Trocha SD, Bilchik AJ: Colorectal carcinoma nodal staging. Frequency and nature of cytokeratin - positive cells in sentinel and nonsentinel lymph nodes. Arch Pathol Lab Med 2003, 1 27:673-679.

69. Trocha SD, Nora DT, Saha SS, Morton DL, Wiese D, Bilchik AJ: Combination probe and dye-directed lymphatic mapping detects micrometastases in early colorectal cancer. J Gastrointest Surg 2003, 7:340-345.

70. Viehl CT, Hamel CT, Marti WR, Guller U, Eisner L, Stammberger U, Terracciano L, Spichtin HP, Harder F, Zuber M: Identification of sentinel lymph nodes in colon cancer depends on the amount of dye injected relative to tumor size. World J Surg 2003, 27: $1285-1290$.

7I. Levine EA, Shen P, Shiver SA, Waters G, Brant A, Geisenger KR: Intraoperative imprint cytology for evaluation of sentine lymph nodes from visceral malignancies. I Gastrointest Surg 2003, 7:687-691.

72. Saha S, Monson KM, Bilchik A, Beutler T, Dan AG, Schochet E, Wiese D, Kaushal S, Ganatra B, Desai D: Comparative analysis of nodal upstaging between colon and rectal cancers by sentinel lymph node mapping: a prospective trial. Dis Colon Rectum 2004, 47: 1767-1772

73. Dan AG, Saha S, Monson KM, Wiese D, Schochet E, Barber KR, Ganatra $B$, Desai $D$, Kaushal S: I\% lymphazurin vs $10 \%$ fluorescein for sentinel node mapping in colorectal tumors. Arch Surg 2004, 139: I 180-1184.

74. Bertoglio S, Sandrucci S, Percivale P, Goss M, Gipponi M, Moresco L, Mussa $B$, Mussa $A$ : Prognostic value of sentinel lymph node biopsy in the pathologic staging of colorectal cancer patients. J Surg Oncol 2004, 85: I66-170.

75. Read TE, Fleshman JW, Caushaj PF: Sentinel lymph node mapping for adenocarcinoma of the colon does not improve staging accuracy. Dis Colon Rectum 2005, 48:80-85.

76. Patten LC, Berger DH, Rodriguez-Bigas M, Mansfield P, Delpassand E, Cleary KR, Fagan SP, Curley SA, Hunt KK, Feig BW: A prospective evaluation of radiocolloid and immunohistochemical staining in colon carcinoma lymphatic mapping. Cancer 2004, 100:2104-2109.

77. Bertagnolli M, Miedema B, Redston M, Dowell J, Niedzwiecki D, Fleshman J, Bem J, Mayer R, Zinner M, Compton C: node staging of resectable colon cancer: results of a multicenter study. Ann Surg 2004, 240:624-628.

78. Saha S, Dan AG, Berman B, Wiese D, Schochet E, Barber K, Choudhr S, Kaushal S, Ganatra B, Desai D, Nagaraju M, Mannam S: Lymphaz- urin $\mathrm{I} \%$ versus $99 \mathrm{mT}$ c sulfur colloid for lymphatic mapping in colorectal tumors: a comparative analysis. Ann Surg Oncol 2004, I I:2I-26.

79. Saha S, Dan AG, Beutler T, Wiese D, Schochet E, Badin J, Branigan T, $\mathrm{Ng}$ P, Bassily N, David D: Sentinel lymph node mapping technique in colon cancer. Semin Oncol 2004, 31 :374-38I.

80. Bembenek A, Schneider U, Gretschel S, Fischer J, Schlag PM: Detection of lymph node micrometastases and isolated tumor cells in sentinel and nonsentinel lymph nodes of colon cancer patients. World J Surg 2005, 29: I I72-I I75.

81. Codignola C, Zorzi F, Zaniboni A, Mutti S, Rizzi A, Padolecchia E, Morandi GB: Is there any role for sentinel node mapping in colorectal cancer staging? Personal experience and review of the literature. Jpn J Clin Oncol 2005, 35:645-650.

82. Dahl K, Westlin J, Kraaz W, Winqvist O, Bergkvist L, Thörn M: Identification of sentinel nodes in patients with colon cancer. Eur J Surg Oncol 2005, 3 I:38I-385.

83. Bilchik AJ, DiNome M, Saha S, Turner RR, Wiese D, McCarter M, Hoon DS, Morton DL: Prospective multicenter trial of staging adequacy in colon cancer: preliminary results. Arch Surg 2006 , | 4 I:527-533.

84. Tuech JJ, Pessaux P, Di Fiore F, Nitu V, Lefebure B, Colson A, Michot $F$ : Sentinel node mapping in colon carcinoma: in-vivo versus ex-vivo approach. Eur J Surg Oncol 2006, 32:158-161.

85. Saha S, Seghal R, Patel M, Doan K, Dan A, Bilchik A, Beutler T, Wiese $D$, Bassily N, Yee C: A multicenter trial of sentinel lymph node mapping in colorectal cancer: prognostic implications for nodal staging and recurrence. Am J Surg 2006, I 91:305-3 I0.

86. Kelder W, Berg A van den, Leij J van der, Bleeker W, Tiebosch AT, Grond JK, Baas PC, Plukker JT: RT-PCR and immunohistochemical evaluation of sentinel lymph nodes after in vivo mapping with Patent Blue V in colon cancer patients. Scand J Gastroenterol 2006, 41: 1073-108.

87. Thomas KA, Lechner J, Shen P, Waters GS, Geisinger KR, Levine EA Use of sentinel node mapping for cancer of the colon: 'to map or not to map". Am Surg 2006, 72:606-6II.

88. Covarelli P, Cristofani R, Boselli C, Servoli A, Burattini MF, Badolato $M$, Cini C, Finocchi L, Noya G: Preliminary study on radioguided sentinel node identification in colon cancer. Am Surg 2007 73:222-226.

89. Kelder W, Berg A van den, Leij J van der, Bleeker W, Tiebosch AT, Grond JK, Baas PC, Plukker JT: RT-PCR and immunohistochemical evaluation of sentinel lymph nodes after in vivo mapping with Patent Blue $\mathbf{V}$ in colon cancer patients. Scand J Gastroenterol 2006, 4I: 1073-1078.

90. Bianchi PP, Ceriani C, Rottoli M, Torzilli G, Roncalli M, Spinelli A, Montorsi M: Laparoscopic lymphatic mapping and sentinel lymph node detection in colon cancer: technical aspects and preliminary results. Surg Endosc 2007, 2 I: I567-157|

91. Murawa D, Filas V, Breborowicz J, Spychała A, Dworzecka K, Murawa $P$ : Evaluation of the sentinel node biopsy in colorectal carcinoma including the results of immunohistochemical examinations. Acta Chir Belg 2007, 107:45-48.

92. Bembenek AE, Rosenberg R, Wagler E, Gretschel S, Sendler A, Siewert JR, Nährig J, Witzigmann H, Hauss J, Knorr C, Dimmler A, Gröne J, Buhr HJ, Haier J, Herbst H, Tepel J, Siphos B, Kleespies A, Koenigsrainer A, Stoecklein NH, Horstmann O, Grützmann R, Imdahl A Svoboda D, Wittekind C, Schneider W, Wernecke KD, Schlag PM: Sentinel lymph node biopsy in colon cancer: a prospective multicenter trial. Ann Surg 2007, 245:858-863.

93. Sandrucci S, Mussa B, Goss M, Mistrangelo M, Satolli MA, Sapino A Bellò M, Bisi G, Mussa A: Lymphoscintigraphic localization of sentinel node in early colorectal cancer: results of a monocentric study. I Surg Oncol 2007, 96:464-469.

94. Tiffet $O$, Kaczmarek $D$, Chambonnière $M L$, Guillan $T$, Baccot $S$, Prévot N, Bageacu S, Bourgeois E, Cassagnau E, Lehur PA, Dubois F: Combining radioisotopic and blue-dye technique does not improve the false-negative rate in sentinel lymph node mapping for colorectal cancer. Dis Colon Rectum 2007, 50:962-970.

95. Lim SJ, Feig BW, Wang H, Hunt KK, Rodriguez-Bigas MA, Skibber JM, Ellis V, Cleary K, Chang G]: Sentinel Lymph Node Evaluation Does Not Improve Staging Accuracy in Colon Cancer. Ann Surg Oncol 2008, I5:46-5I.

96. Kusano M, Tajima Y, Yamazaki K, Kato M, Watanabe M, Miwa M: Sentinel node mapping guided by indocyanine green fluores- 
cence imaging: a new method for sentinel node navigation surgery in gastrointestinal cancer. Dig Surg 2008, 25: $103-108$.

97. Quadros CA, Lopes A, Araujo I, Fregnani JH, Fahel F: Upstaging benefits and accuracy of sentinel lymph node mapping in colorectal adenocarcinoma nodal staging. J Surg Oncol 2008, 98:324-330.

98. Higgins JPT, Green S, editors: Cochrane Handbook for Systematic Reviews of Interventions 4.2.6 [updated September 2006]. In The Cochrane Library Issue 4 Chichester, UK: John Wiley \& Sons, Ltd; 2006.

99. Redston M, Compton CC, Miedema BW, Niedzwiecki D, Dowell JM, Jewell SD, Fleshman JM, Bem J, Mayer RJ, Bertagnolli MM, Leukemia Group B Trial 80001: Analysis of micrometastatic disease in sentinel lymph nodes from resectable colon cancer: results of Cancer and Leukemia Group B Trial 8000I. J Clin Oncol 2006, 24:878-883.

100. Sticca RP: Is there clinical value to sentinel lymph node sampling in colon cancer. I Clin Oncol 2006, 24:84 I-842.

10I. Pocard M, Eynde M Van den, Goere D, Boige V, Malka D: Sentinel lymph node sampling and analysis in colon cancer: what is the question? J Clin Oncol 2006, 24:37I2-37I3. author reply 37I33714

102. Ellis LM: A perspective on sentinel lymph node biopsy in colorectal cancer: the race between surgical technology and molecular oncology. Ann Surg Oncol 2000, 7:475-476.

103. Bilchik AJ, Nora DT: Lymphatic mapping of nodal micrometastasis in colon cancer: putting the cart before the horse? Ann Surg Oncol 2002, 9:529-53I.

104. Brennan DJ, Moynagh M, Brannigan AE, Gleeson F, Rowland M, O'Connell PR: Routine mobilization of the splenic flexure is not necessary during anterior resection for rectal cancer. Dis Colon Rectum 2007, 50:302-307.

105. Sarli L, Cinieri FG, Pavlidis C, Regina G, Sansebastiano G, Veronesi L, Ferro $M$, Violi $V$, Roncoroni L: Anorectal function problems after left hemicolectomy. J Laparoendosc Adv Surg Tech 2006, 16:565-57|.

106. Sarli L, Pavlidis C, Cinieri FG, Regina G, Sansebastiano G, Veronesi L, Ferro M, Morari S, Violi V, Roncoroni L: Prospective comparison of laparoscopic left hemicolectomy for colon cancer with laparoscopic left hemicolectomy for benign colorectal disease. World J Surg 2006, 30:446-452.

107. Rouffet F, Hay JM, Vacher B, Fingerhut A, Elhadad A, Flamant $Y$ Mathon C, Gainant A: Curative resection for left colonic carcinoma: hemicolectomy vs. segmental colectomy. A prospective, controlled, multicenter trial. French Association for Surgical Research. Dis Colon Rectum 1994, 37:65I-659.

108. Ho YH, Low D, Goh HS: Bowel function survey after segmental colorectal resections. Dis Colon Rectum 1996, 39:307-310.

109. Busuttil RW, Foglia RP, Longmire WP Jr: Treatment of carcinoma of the sigmoid colon and upper rectum. A comparison of local segmental resection and left hemicolectomy. Arch Surg 1 977, I | 2:920-923.

1 10. Chen SL, Bilchik A): More extensive nodal dissection improves survival for stages I to III of colon cancer. Ann Surg 2006, 244:602-610.

III. Steele G]: Colorectal cancer. Edited by: McKenna RJ, Murphy GP. Cancer surgery, Philadelphia: Lippincott; 1994:125-84.

I I2. Joseph NE, Sigurdson ER, Hanlon AL, Wang H, Mayer RJ, MacDonald JS, Catalano PJ, Haller DG: Accuracy of determining nodal negativity in colorectal cancer on the basis of the number of nodes retrieved on resection. Ann Surg Oncol 2003, 10:2/3-218.

1 13. Trocha S, Krasne D, Morton DL: Effect of lymphatic mapping on the new tumor-node-metastasis classification for colorectal cancer. I Clin Oncol 2003, $21: 668-72$.

114. Des Guetz G, Uzzan B, Nicholas P, Cucherat M, de Mestier P, Morere $J F$, Breau JL, Perret $G$ : Is sentinel lymph node mapping in colorectal cancer a future prognostic factor? A meta-analysis. World J Surg 2007, 3 I: 1304-1312.

1 15. Bilchik AJ, Hoon DS, Saha S, Turner RR, Wiese D, DiNome M, Koyanagi K, McCarter M, Shen P, Iddings D, Chen SL, Gonzalez M, Elashoff $D$, Morton DL: Prognostic impact of micrometastases in colon cancer. Ann Surg 2007, 246:568-575.

I 16. Borgstein PJ, Pijpers R, Comans EF, van Diest PJ, Boom RP, Meijer S: Sentinel lymph node biopsy in breast cancer: guidelines and pitfalls of lymphoscintigraphy and gamma probe detection. Am Coll Surg 1998, 186:275-283.
117. Cox CE, Pendas S, Cox JM, Joseph E, Shons AR, Yeatman T, Ku NN, Lyman GH, Berman C, Haddad F, Reintgen DS: Guidelines for sentinel node biopsy and lymphatic mapping of patients with breast cancer. Ann Surg 1998, 227:645-653.

I 18. O'Hea BJ, Hill AD, El-Shirbiny AM, Yeh SD, Rosen PP, Coit DG, Borgen PI, Cody HS 3rd: Sentinel lymph node biopsy in breast cancer: initial experience at Memorial Sloan-Kettering Cancer Center. J Am Coll Surg 1998, 186:423-427.

II9. Veronesi U, Zurrida S, Galimberti V: Consequences of sentinel node in clinical decision making in breast cancer and prospects for future studies. Eur J Surg Oncol 1998, 24:93-95.

120. Hutchinson JR, Chagpar AB, Scoggins CR, Martin RC 2nd, Carlson DJ, Laidley AL, El-Eid SE, McGlothin TQ, Noyes RD, Ley PB, Tuttle TM, McMasters KM, University of Louisville Breast Sentinel Lymph Node Study: Surgeon and community factors affecting breast cancer sentinel lymph node biopsy. Am J Surg 2005, 190:903-906.

12I. Feezor RJ, Copeland EM, Hochwald SN: Significance of micrometastases in colorectal cancer. Ann Surg Oncol 2002, 9:944-953.

122. Ota DM: Is intraoperative lymph node mapping and sentinel node biopsy for colorectal carcinoma necessary? Ann Surg Oncol 2000, 7:82-84.

123. Miyashiro I, Miyoshi N, Hiratsuka M, Kishi K, Yamada T, Ohue M, Ohigashi $\mathrm{H}$, Yano M, Ishikawa O, Imaoka S: Detection of sentinel node in gastric cancer surgery by indocyanine green fluorescence imaging: comparison with infrared imaging. Ann Surg Oncol 2008, I5:1640-3.

124. Cody HS 3rd, Fey J, Akhurst T, Fazzari M, Mazumdar M, Yeung H, Yeh $\mathrm{SD}$, Borgen PI: Complementarity of blue dye and isotope in sentinel node localization for breast cancer: univariate and multivariate analysis of $\mathbf{9 6 6}$ procedures. Ann Surg Oncol 200I, 8:13-19.

125. Gershenwald JE, Tseng CH, Thompson W, Mansfield PF, Lee JE, Bouvet M, Lee JJ, Ross MI: Improved sentinel lymph node localization in patients with primary melanoma with the use of radiolabeled colloid. Surgery 1998, I 24:203-210

126. Albertini JJ, Cruse CW, Rapaport D, Wells K, Ross M, DeConti R, Berman CG, Jared K, Messina J, Lyman G, Glass F, Fenske N, Reintgen DS: Intraoperative radio-lympho-scintigraphy improves sentinel lymph node identification for patients with melanoma. Ann Surg 1996, 223:217-224

127. Hunerbein M, Totkas S, Ghadimi BM, Schlag PM: Preoperative evaluation of colorectal neoplasms by colonoscopic miniprobe ultrasonography. Ann Surg 2000, 232:46-50.

128. Tew K, Irwig L, Matthews A, Crowe P, Macaskill P: Meta-analysis of sentinel node imprint cytology in breast cancer. Br J Surg 2005, 92:1068-1080.

129. Ali R, Hanly AM, Naughton P, Castineira CF, Landers R, Cahill RA, Watson RGK: Intraoperative frozen section assessment of sentinel lymph nodes in the operative management of women with symptomatic breast cancer. World J Surg Oncol 2008, 6:69.

130. Tsujimoto M, Nakabayashi K, Yoshidome K, Kaneko T, Iwase T, Akiyama $F$, Kato $Y$, Tsuda $H$, Ueda $S$, Sato $K$, Tamaki $Y$, Noguchi $S$, Kataoka TR, Nakajima H, Komoike Y, Inaji H, Tsugawa K, Suzuki K, Nakamura S, Daitoh M, Otomo Y, Matsuura N: One-step nucleic acid amplification for intraoperative detection of lymph node metastasis in breast cancer patients. Clin Cancer Res 2007 , 13:4807-48|6.

13I. Hughes SJ, Xi L, Raja S, Gooding W, Cole DJ, Gillanders WE, Mikhitarian K, McCarty K, Silver S, Ching J, McMillan W, Luketich JD, Godfrey TE: A rapid, fully automated, molecular-based assay accurately analyzes sentinel lymph nodes for the presence of metastatic breast cancer. Ann Surg 2006, 243:389-398.

132. Hama Y, Koyama Y, Urano Y, Choyke PL, Kobayashi H: Simultaneous two-color spectral fluorescence lymphangiography with near infrared quantum dots to map two lymphatic flows from the breast and the upper extremity. Breast Cancer Res Treat 2007, 103:23-28.

133. Johnson KS, Chicken DW, Pickard DC, Lee AC, Briggs G, Falzon M, Bigio IJ, Keshtgar MR, Brown SG: Elastic scattering spectroscopy for intraoperative determination of sentinel lymph node status in the breast. J Biomed Opt 2004, 9: I | 22- I I 28.

134. Yamashita K, Sakuramoto S, Kikuchi S, Katada N, Kobayashi N, Watanabe M: Validation of staging systems for gastric cancer. Gastric Cancer 2008, I I: I I - I I8. 
135. O'Connell JB, Maggard MA, Ko CY: Colon cancer survival rates with the new American Joint Committee on Cancer sixth edition staging. J Nat/ Cancer Inst 2004, 96: | 420- I 425.

136. Gunderson LL, Jessup JM, Sargent DJ, Greene FL, Stewart A, for AJCC 7th Ed'n Hindgut Taskforce (HTF): TN categorization for rectal and colon cancers based on national survival outcome data. J Clin Oncol 2008, 26:. (May 20 suppl; abstr 4020)

\section{Pre-publication history}

The pre-publication history for this paper can be accessed here:

http://www.biomedcentral.com/1471-2482/8/17/prepub

Publish with Bio Med Central and every scientist can read your work free of charge

"BioMed Central will be the most significant development for disseminating the results of biomedical research in our lifetime. "

Sir Paul Nurse, Cancer Research UK

Your research papers will be:

- available free of charge to the entire biomedical community

- peer reviewed and published immediately upon acceptance

- cited in PubMed and archived on PubMed Central

- yours - you keep the copyright

Submit your manuscript here:

http://www.biomedcentral.com/info/publishing_adv.asp
BioMedcentral 